\title{
Nonthermal dark matter via Affleck-Dine baryogenesis and its detection possibility
}

\author{
Masaaki Fujii \\ Department of Physics, University of Tokyo, Tokyo 113-0033, Japan \\ and CERN Theory Division, CH-1211 Geneva 23, Switzerland \\ K. Hamaguchi \\ Department of Physics, Tohoku University, Sendai 980-8578, Japan
}

(Received 20 May 2002; published 3 October 2002)

\begin{abstract}
The formation and late time decays of $Q$ balls are generic consequences of the Affleck-Dine (AD) baryogenesis. A substantial number of the lightest supersymmetry (SUSY) particles (LSPs) are produced nonthermally as the decay products of these $Q$ balls. This requires a significantly large annihilation cross section of the LSP so as not to overclose the universe, which predicts a Higgsino- or $W$-ino-like LSP instead of the standard $B$-ino LSP. We have reexamined the AD baryogenesis with special attention to the late-time decays of the $Q$ balls, and then specified the parameter regions where the LSPs produced by the $Q$ ball decays result in a cosmologically interesting mass density of dark matter by adopting several SUSY breaking models. This reveals new cosmologically interesting parameter regions, which have not attracted much attention so far. We have also investigated the prospects of direct and indirect detection of these dark matter candidates, and found that there is an intriguing possibility to detect them in various next generation dark matter searches.
\end{abstract}

DOI: 10.1103/PhysRevD.66.083501

PACS number(s): 95.35. $+\mathrm{d}, 12.60 . \mathrm{Jv}, 98.80 . \mathrm{Cq}$

\section{INTRODUCTION}

Both the origin and nature of dark matter and the production mechanism of the baryon (matter-antimatter) asymmetry in the present universe are among the most fundamental puzzles in cosmology and in particle physics. Of the many candidates for dark matter, the lightest supersymmetric particle (LSP) has been attracting wide interest as an ideal candidate, which is inherent in the supersymmetric (SUSY) standard model, and it is exactly stable under $R$-parity conservation.

On the other hand, once we introduce SUSY in the standard model, there appear a number of flat directions in the scalar potential carrying baryon $(B)$ and/or lepton $(L)$ number. Affleck and Dine (AD) proposed an interesting mechanism [1] to generate effectively a baryon asymmetry by using these flat directions. Because of the flatness of the potential, it is quite plausible that a linear combination of squark and/or slepton fields has a large expectation value along a flat direction during the inflation in the early universe. After the end of inflation, this flat direction field $\phi$ starts a coherent oscillation. At this stage, because of baryon (and/or lepton-) number-violating operators in the scalar potential, the $\phi$ field gets a nonzero motion along the phase direction as well, which means nothing but baryon (and/or lepton) number generation since the $\phi$ carries a baryon (and/or lepton) number.

Recently, we have pointed out that the Higgsino- or $W$-ino-like neutralino naturally becomes the dominant component of the dark matter, if either of them is the LSP and if the $\mathrm{AD}$ mechanism is responsible for the generation of the observed baryon asymmetry in the present universe [2]. ${ }^{1}$ The

\footnotetext{
${ }^{1}$ We do not consider the gauge-mediated SUSY breaking models [3], where the $Q$ ball is generally stable [4,5].
}

LSP is produced nonthermally by the late-time decay of the $Q$ balls, which are generally formed in the AD mechanism [5-7]. Then, the large pair annihilation cross section of the Higgsino- or $W$-ino-like LSP leads to the desired mass density of dark matter, instead of overclosing the universe, as in the case of the standard $B$-ino LSP. It should be noticed that the typical decay temperature of the $Q$ ball is between $1 \mathrm{MeV}$ and (a few) $\mathrm{GeV}$, i.e. below the freeze-out temperature of the LSP and before the big-bang nucleosynthesis, which is just the desired range for the present scenario. In this scenario, it is very interesting that both the dark matter and the baryon asymmetry are produced by a single source, the $Q$ ball decay, which originates from the AD condensation. Furthermore, the relatively strong interactions of these LSPs significantly enlarge the possibility of detecting these dark matter candidates in both direct and indirect searches.

In this paper, we give more detailed analyses of this scenario, "Higgsino or $W$-ino dark matter from $Q$ ball decay," adopting several SUSY breaking models, which include the minimal supergravity (MSUGRA) scenario, the anomalymediated SUSY breaking (AMSB) model [8] with additional universal scalar mass, and the no-scale model with nonuniversal gaugino masses [9]. In each model, we will show parameter regions in which the LSP from the $Q$ ball decay can be the dominant component of the dark matter. This predicts new cosmologically interesting parameter regions, which have not attracted much attention; the $W$-ino dark matter is realized in wide parameter regions in the AMSB models and the no-scale models, where the nonthermal $W$-ino production via decays of $Q$ balls naturally explains the mass density of the dark matter. Even in the MSUGRA scenario, Higgsino dark matter is realized in a relatively wide region by virtue of the "focus point" behavior of $m_{H_{u}}^{2}$ [10], in which the mass density of dark matter is explained by the nonthermally produced Higgsino. 
We also investigate the direct and indirect detection of the neutralino dark matter in these regions. We calculate the proton-neutralino scalar cross section for the direct detection. As for the indirect detection, we adopt the $\chi \chi \rightarrow \gamma \gamma$ annihilation channel, which produces monoenergetic $\gamma$-ray lines. We estimate the neutralino annihilation rate into the $2 \gamma$ final state using the full one-loop calculations presented in Ref. [11], and discuss the possibility of detection in the next generation of air Cherenkov telescopes for several models of density profile in the halo. Actually, the large Higgsino or $W$-ino content of the LSP strongly enhances the direct and indirect detection rates, via the Higgs exchange diagrams and the chargino- $W$ boson loop diagrams, respectively. As we will see, if the Higgsino or $W$-ino is really a significant component of dark matter, there exists an intriguing possibility to detect these nonthermal dark matter candidates in various next-generation detectors.

Note that, if the Higgsino- or $W$-ino-like dark matter is indeed detected at future experiments, it suggests the existence of nonthermal sources for these LSPs, which predicts a highly nontrivial history of the universe. This might discriminate the origin of the baryon asymmetry between various scenarios.

The rest of this paper is organized as follows: first, we reexamine the $\mathrm{AD}$ baryogenesis scenario, giving particular attention to the late-time decay of $Q$ balls in Sec. II. This includes an interesting new twist in the $\mathrm{AD}$ baryogenesis using nonrenormalizable operators in the Kähler potential to rotate the $\mathrm{AD}$ field. This scenario is now able to explain the observed baryon asymmetry without any assumptions of additional large entropy production to dilute the resultant baryon asymmetry, such as decays of heavy moduli fields and/or a thermal inflation. In Sec. III, we review the properties of the $Q$ ball in the minimal SUSY standard model (MSSM). We discuss the nonthermal production of the LSP dark matter from the $Q$ ball decay in Sec. IV. Our main results are given in Sec. V. We show in several SUSY breaking models the parameter spaces where the Higgsino- and $W$-ino-like LSPs from the $Q$ ball decay can be the dominant component of the dark matter, and we investigate their direct and indirect detection in detail. We also comment on the possibility that the nonthermally produced neutralino via the late-time decays of $Q$ balls forms the warm dark matter, which erases the cuspy profile of the matter density of the halo that might be inconsistent with the observations. We present concluding remarks and discussions in Sec. VI.

\section{AFFLECK-DINE MECHANISM}

In this section we briefly review the Affleck-Dine mechanism $[1,12]$ in the MSSM. Hereafter, we assume $R$-parity conservation and the following superpotential at renormalizable level as usual:

$$
W=y_{U} Q \bar{U} H_{u}+y_{D} Q \bar{D} H_{d}+y_{E} L \bar{E} H_{d}+\mu H_{u} H_{d} .
$$

Here, $Q, \bar{U}, \bar{D}, L, \bar{E}, H_{u}$ and $H_{d}$ denote superfields of lefthanded quark doublets, right-handed up-type and down-type quarks, left-handed lepton doublets, right-handed charged leptons and Higgs doublets, respectively; we omit the family indices for simplicity. In the scalar potential, there are a number of flat directions along which the $F$-term potential coming from the superpotential in Eq. (1) as well as the $D$-term potential vanish [13]:

$$
\begin{gathered}
L H_{u}, H_{u} H_{d}, \\
\bar{U} \bar{D} \bar{D}, L L \bar{E}, Q \bar{D} L, \\
Q Q Q L, \bar{U} \bar{U} \bar{D} \bar{E}, Q \bar{U} Q \bar{D}, Q \bar{U} L \bar{E}, \cdots,
\end{gathered}
$$

where the ellipsis denotes the flat directions consisting of linear combinations with more scalar fields. In the following, we will parametrize a flat direction by a complex scalar field $\phi$.

\section{A. Affleck-Dine mechanism with nonrenormalizable operators in the superpotential}

In the supersymmetric limit, the flat directions are lifted only by nonrenormalizable operators in the scalar potential. Let us first assume that all the nonrenormalizable operators consistent with the MSSM gauge symmetry and the $R$-parity exist in the superpotential. Most of the flat directions are then lifted by the scalar potential coming from the following dimension-4 superpotentials:

$$
W=\frac{\lambda}{M} L H_{u} L H_{u}, \frac{\lambda}{M} Q Q Q L, \frac{\lambda}{M} \bar{U} \bar{U} \bar{D} \bar{E},
$$

where $M$ denotes effective scales at which these nonrenormalizable operators are induced. Here and hereafter, we discard superpotentials that conserve the baryon and lepton numbers (e.g. $W \propto Q \bar{U} Q \bar{D}, Q \bar{U} L \bar{E}$ ), since they cannot generate baryon asymmetry. We have included superpotentials conserving $B-L$, since the $Q$ ball can decay after the electroweak phase transition and the baryon asymmetry is not washed out by the sphaleron effect [14].

Some of remaining flat directions are lifted by the following dimension-6 superpotentials:

$$
W=\frac{\lambda}{M^{3}} \bar{U} \bar{D} \bar{D} \bar{U} \bar{D} \bar{D}, \frac{\lambda}{M^{3}} L L \bar{E} L L \bar{E}
$$

All the other flat directions are lifted either by $B$ - and $L$ conserving superpotentials or by superpotentials of the form $W=\left(\lambda / M^{n-3}\right) \psi \phi^{n-1}[13]$. In the latter case, one can show that $\psi$ becomes zero when the $\phi$ field develops a large vacuum expectation value. Therefore, this type of super- 
potential cannot provide a nonzero $A$-term that is indispensable to create a net baryon/lepton asymmetry as will be discussed soon. Thus, in the following, we write the superpotential that lifts a flat direction $\phi$ as

$$
W=\frac{1}{n M^{n-3}} \phi^{n},
$$

with $n=4$ or 6 . Note that we have normalized the effective scale $M$ including the coupling $\lambda$, and hence $M$ can be larger than the cut-off scale (e.g. Planck scale).

In the case of the leptonic flat direction (e.g. $L H_{u}, L L \bar{E}$ ), lepton asymmetry is first generated by the AD mechanism. Then a part of the produced lepton asymmetry is converted [15] into baryon asymmetry through the sphaleron effect. In particular, leptogenesis via the $L H_{u}$ flat direction [16] has attracted much attention [17-22], since in this case the baryon asymmetry in the present universe is directly related to the neutrino mass. This allows us to obtain definite predictions on the rate of the neutrinoless double beta decay $[19,21]$. In this paper, however, we do not consider the $L H_{u}$ flat direction since the resultant $Q$ ball along this direction is very small and evaporates well above the weak scale $[6,23]$. As for the other leptonic flat directions, large $Q$ balls can be formed. However, the $Q$ balls must decay or evaporate well before the sphaleron effect terminates $(T \sim 100 \mathrm{GeV})$, which requires some mechanisms to make $Q$ balls small enough $[24,25]$. In this case, the LSPs produced by the decays of $Q$ balls are thermalized and the $B$-ino-like LSP is the almost unique candidate for neutralino dark matter. We do not treat such cases in the following discussions, and concentrate on the flat directions carrying a nonzero baryon number.

Let us now discuss the $\mathrm{AD}$ mechanism with a nonrenormalizable superpotential. The relevant scalar potential for $\phi$ is given by

$$
\begin{aligned}
V(\phi)= & \left(m_{\phi}^{2}-c_{H} H^{2}\right)|\phi|^{2}+\frac{m_{3 / 2}}{n M^{n-3}}\left(a_{m} \phi^{n}+\text { H.c. }\right) \\
& +\frac{1}{M^{2 n-6}}|\phi|^{2 n-2} .
\end{aligned}
$$

Here, the potential terms, which are proportional to the soft mass squared $m_{\phi}^{2}$ and the gravitino mass $m_{3 / 2}$, come from the SUSY breaking at the true vacuum. We will concentrate on gravity-mediated and gaugino-mediated SUSY breaking models, and take $m_{\phi} \simeq m_{3 / 2}\left|a_{m}\right| \simeq 1 \mathrm{TeV}$, hereafter. (We will discuss later the case of anomaly-mediated SUSY breaking models, where $m_{3 / 2} \gg m_{\phi}$.) The terms depending on the Hubble parameter $H$ denote the effect of SUSY breaking caused by the finite energy density of the inflaton [12]: $c_{H}$ is a real constant of order unity, which depends on the couplings between the inflaton and the $\phi$ field in the Kähler potential. Hereafter, we take $c_{H} \simeq 1(>0)$, which is crucial to let $\phi$ have a large expectation value during inflation. There might also exist a Hubble-induced $A$-term potential, which has the same form as the second term in Eq. (6), with
$H$ instead of $m_{3 / 2} \cdot{ }^{2}$ Even in the presence of this term, the following discussions are not altered, ${ }^{3}$ so we assume its absence in the present work, for simplicity. The last term is the $F$-term potential coming from the superpotential in Eq. (5). Here, we assume the absence of thermal effects, which will be justified later.

The estimation of the baryon asymmetry is rather straightforward. The baryon number density is related to the $\mathrm{AD}$ field as

$$
n_{B}=\beta i\left(\dot{\phi}^{*} \phi-\phi^{*} \dot{\phi}\right)
$$

where $\beta$ is the corresponding baryon charge of the AD field, which is at most $1 / 3$. The equation of motion for the AD field in the expanding universe is given by

$$
\ddot{\phi}+3 H \dot{\phi}+\frac{\partial V}{\partial \phi^{*}}=0
$$

Then, together with Eq. (7), the equation of motion for the baryon number density is written as follows:

$$
\begin{aligned}
\dot{n}_{B}+3 H n_{B} & =2 \beta \operatorname{Im}\left(\frac{\partial V}{\partial \phi} \phi\right) \\
& =2 \beta \frac{m_{3 / 2}}{M^{n-3}} \operatorname{Im}\left(a_{m} \phi^{n}\right),
\end{aligned}
$$

where we take $m_{3 / 2}$ as real by adjusting the phase of $a_{m}$. One can see that the production rate of the baryon number is proportional to the $A$-term. By integrating this equation, we obtain the baryon number at the cosmic time $t$ as

$$
\left[R^{3} n_{B}\right](t)=2 \beta \frac{\left|a_{m}\right| m_{3 / 2}}{M^{n-3}} \int^{t} R^{3}|\phi|^{n} \sin \theta d t,
$$

where $R$ is the scale factor of the expanding universe, and $\theta \equiv \arg \left(a_{m}\right)+n \arg (\phi)$.

During inflation, the negative Hubble mass term $-c_{H} H^{2}|\phi|^{2}$ causes an instability of the flat direction field $\phi$ around the origin, and the $\mathrm{AD}$ field acquires a large expectation value:

$$
|\phi| \simeq\left(H_{I} M^{n-3}\right)^{1 /(n-2)}
$$

\footnotetext{
${ }^{2}$ This requires the existence of a three-point coupling of the AD field to the inflaton in the Kähler potential, $\delta K \supset I \phi^{\dagger} \phi / M_{p l}$ + H.c., where $I$ denotes the inflaton superfield. This coupling leads to a relatively high reheating temperature of the inflation, $T_{R}$ $\sim m_{I}\left(m_{I} / M_{p l}\right)^{1 / 2}$, where $m_{I}$ is the mass of the inflaton. Therefore, as we will see, the absence of this three-point coupling is also desirable for obtaining the low reheating temperature that explains the right amount of baryon asymmetry. Notice that $c_{H} \simeq 1$ does not need this three-point coupling, which is consistent with the low reheating temperatures.

${ }^{3}$ See the related discussions in Refs. [12,17,19].
} 
where $H_{I}$ denotes the Hubble parameter during inflation. This is the balance point between the negative Hubble mass term $-c_{H} H^{2}|\phi|^{2}$ and the $F$-term potential $|\phi|^{2 n-2} / M^{2 n-6}$. Note that the curvature along the phase direction $m_{p h}^{2}$ is much smaller than $H_{I}^{2}$ at this point, since

$$
m_{p h}^{2} \simeq \frac{m_{3 / 2}}{M^{n-3}}|\phi|^{n-2} \simeq m_{3 / 2} H_{I} \ll H_{I}^{2} .
$$

Therefore, the initial phase of the AD field fixed during inflation does not generally coincide with the minimum of the $A$-term potential in Eq. (6), and hence we naturally expect that $\sin \theta=\mathcal{O}(1)$.

After the end of inflation, the AD field slowly rolls down toward the origin following the gradual decrease of the Hubble parameter $H$ as $|\phi(t)| \simeq\left[H(t) M^{n-3}\right]^{1 / n-2}$. At this slow rolling regime, the right-hand side of Eq. (10) increases as $\propto t^{2-2 /(n-2)}$. Here, we have assumed the matterdominated universe, which is true as long as $T_{R} \lesssim 2$ $\times 10^{10} \mathrm{GeV}\left(m_{\phi} / 10^{3} \mathrm{GeV}\right)^{1 / 2}$. When $H\left(t_{\text {osc }}\right) \simeq m_{\phi}$, the soft mass term of the $\mathrm{AD}$ field eventually dominates the negative Hubble mass term, and causes the coherent oscillation of the AD field around the origin. After this time, the amplitude of the AD field rapidly decreases as $|\phi| \propto t^{-1}$, and then the production of the baryon number terminates at the time $H_{\text {osc }}$ $\simeq m_{\phi}$.

Using the above arguments and Eq. (10), we obtain the baryon number density at the time $t=t_{\mathrm{osc}}$ :

$$
\begin{aligned}
n_{B}\left(t_{\mathrm{osc}}\right)= & \frac{2(n-2)}{3(n-3)} \beta \delta_{\mathrm{eff}}\left|a_{m}\right| m_{3 / 2} \\
& \times\left(H_{\mathrm{osc}} M^{n-3}\right)^{2 /(n-2)},
\end{aligned}
$$

where $\delta_{\text {eff }} \equiv \sin \theta[=\mathcal{O}(1)]$. Then, after completion of the reheating process of the inflation, this leads to the following baryon asymmetry:

$$
\begin{aligned}
\frac{n_{B}}{S} & =\frac{1}{4} \frac{T_{R}}{M_{\mathrm{pl}}^{2} H_{\mathrm{osc}}^{2}} n\left(t_{\mathrm{osc}}\right) \\
& =\frac{n-2}{6(n-3)} \beta \delta_{\mathrm{eff}}\left|a_{m}\right| \frac{m_{3 / 2} T_{R}}{M_{\mathrm{pl}}^{2} H_{\mathrm{osc}}^{2}}\left(H_{\mathrm{osc}} M^{n-3}\right)^{2 /(n-2)},
\end{aligned}
$$

where $T_{R}$ is the reheating temperature of the inflation, $s$ is the entropy density of the universe and $M_{\mathrm{pl}}=2.4$ $\times 10^{18} \mathrm{GeV}$ is the reduced Planck scale. By using $H_{\text {osc }}$ $\simeq m_{\phi}$, it is given by

$$
\frac{n_{B}}{s} \simeq 1 \times 10^{-10} \beta \delta_{\text {eff }}\left|a_{m}\right|\left(\frac{m_{3 / 2}}{m_{\phi}}\right)\left(\frac{M}{M_{\mathrm{pl}}}\right)\left(\frac{T_{R}}{10^{9} \mathrm{GeV}}\right),
$$

for $n=4$, and

$$
\begin{aligned}
\frac{n_{B}}{S} \simeq & 5 \times 10^{-10} \times \beta \delta_{\text {eff }}\left|a_{m}\right|\left(\frac{m_{3 / 2}}{m_{\phi}}\right)\left(\frac{1 \mathrm{TeV}}{m_{\phi}}\right)^{1 / 2} \\
& \times\left(\frac{M}{M_{\mathrm{pl}}}\right)^{3 / 2}\left(\frac{T_{R}}{100 \mathrm{GeV}}\right),
\end{aligned}
$$

for $n=6$. These quantities remain constant unless there are additional entropy productions.

These predictions on the baryon asymmetry are not altered by the presence of the $Q$ ball formation, as long as the produced $Q$ balls are unstable and decay before the big-bang nucleosynthesis (BBN). The details related to the $Q$ balls will be discussed in Sec. III. The only point remaining to be checked is the absence of the thermal effects. In the remainder of this section, we discuss these in the cases of the $n$ $=4$ and $n=6$ superpotentials.

$$
\text { 1. } n=4
$$

In the case of the $n=4$ superpotential, it was pointed out that the thermal effects cause the early oscillations of the $\mathrm{AD}$ field $H_{\mathrm{osc}} \gg m_{\phi}$, which significantly suppress the resultant baryon asymmetry [26]. Analytic and more systematic investigations were done for the case of the leptogenesis via the $L H_{u}$ flat direction, which revealed an interesting property, the "reheating temperature independence" of the baryon asymmetry [19]. For the other flat directions, however, there are some subtleties. The dimension-5 operators coming from the superpotential $\propto Q Q Q L, \bar{U} \bar{U} \bar{D} \bar{E}$ are responsible for the proton decay [27]. Null observations of the proton decay then lead to strong constraints ${ }^{4}$ on the effective cutoff scale, $M \gtrsim 10^{25} \mathrm{GeV}$, at least for the most relevant operators $[28,29]$. Following the analyses in Ref. [19], one can show that there is no thermal effect as long as $T_{R} \leq 10^{6} \mathrm{GeV}$ when $M \geqslant 10^{23} \mathrm{GeV}$, and hence there is no need to worry about the thermal effects in these scenarios.

As we will see, such a large scale of $M$ leads to the formation of very large $Q$ balls with the decay temperature $T_{d}$ $\lesssim 1 \mathrm{GeV}$. Since the decays of the $Q$ balls occur well after the sphaleron effect terminates, the baryon asymmetry is not washed out, although the relevant flat directions conserve the $B-L$ symmetry.

\section{2. $n=6$}

In the case of the $n=6$ superpotential, it is much easier to avoid the thermal effects because the potential is much flatter than in the $n=4$ case. For the appearance of the thermal mass terms, the fields coupled with the $\phi$ field must reside in the thermal bath: $f|\phi|<T$, where $f$ is the Yukawa or gauge coupling constant of the AD field. Then, the sufficient condition to avoid the thermal mass terms is written as

$$
T_{R} \lesssim 10^{8} \mathrm{GeV}\left(\frac{f}{10^{-5}}\right)^{2}\left(\frac{M}{M_{\mathrm{pl}}}\right)^{3 / 2} .
$$

Another thermal effect we have to check comes from the thermal logarithmic potential [30]:

$$
\delta V \supset a T^{4} \log \left(\frac{|\phi|^{2}}{T^{2}}\right),
$$

\footnotetext{
${ }^{4}$ Another possibility is the absence of these operators. In such a case, most of the flat directions are expected to be lifted by the $n$ $=6$ superpotential, and two other operators are operative for AD baryogenesis besides $(\bar{U} \bar{D} \bar{D})^{2}$, such as $\bar{U} \bar{D} \bar{D} Q \bar{D} L, L L \bar{E} \bar{U} \bar{D} \bar{D}$.
} 
where $a$ is a constant given by the fourth power of gauge and/or Yukawa coupling constants. To avoid the early oscillations due to this potential, we need the following condition:

$$
\begin{aligned}
T_{R} & \lesssim \frac{1}{|a|^{1 / 2}} M\left(\frac{m_{\phi}^{3}}{M_{\mathrm{pl}}^{2} M}\right)^{1 / 4} \\
& =7.0 \times 10^{7} \mathrm{GeV}\left(\frac{10^{-2}}{|a|}\right)^{1 / 2}\left(\frac{M}{M_{\mathrm{pl}}}\right)^{3 / 4}\left(\frac{m_{\phi}}{1 \mathrm{TeV}}\right)^{3 / 4},
\end{aligned}
$$

which leads to the comparable constraint on the reheating temperature as Eq. (18). As a result, the early oscillations due to the thermal effects can be easily avoided as long as $T_{R}$ $\lesssim 10^{8} \mathrm{GeV}$ in $\mathrm{AD}$ baryo/leptogenesis with the $n=6$ superpotential.

\section{B. Affleck-Dine mechanism without a superpotential}

The other interesting possibility is that nonrenormalizable operators in the superpotential are forbidden by some chiral symmetries, such as $R$-symmetry. In this case, the initial amplitude of the AD field is determined by the negative Hubble mass term $-c_{H} H^{2}|\phi|^{2}$ and the nonrenormalizable operators in the Kähler potential and is expected to be $|\phi|_{0} \leq M_{\mathrm{pl}}$.

In this section, as an example, we consider the following terms [1], which are consistent with $R$-symmetry:

$$
\delta \mathcal{L}=\int d^{4} \theta\left(\lambda_{1} \frac{Z^{\dagger} Z}{M_{\mathrm{pl}}^{4}} Q Q \bar{U}^{\dagger} \bar{E}^{\dagger}+\lambda_{2} \frac{Z^{\dagger} Z}{M_{\mathrm{pl}}^{4}} Q \bar{U}^{\dagger} \bar{D}^{\dagger} L+\text { H.c. }\right),
$$

where $\lambda_{i}$ 's are coupling constants and $Z$ is the superfield with the nonvanishing $F$-term responsible for the SUSY breaking in the true vacuum. The effective potential of the AD field induced by these terms and SUSY breaking effects is then given by

$$
V=\left(m_{\phi}^{2}-c_{H} H^{2}\right)|\phi|^{2}+\frac{m_{3 / 2}^{2}}{4 M_{\mathrm{pl}}^{2}}\left(\lambda \phi^{4}+\text { H.c. }\right)+\ldots,
$$

where $\lambda$ is a coupling constant and the ellipsis denotes the higher order terms coming from the Kähler potential. (Above the Planck scale the scalar potential is expected to be exponentially lifted by the supergravity effect.)

The evolution of the AD field is much simpler than the case with a nonrenormalizable superpotential. During inflation, the $\mathrm{AD}$ field is fixed at a very large scale $|\phi|_{0} \lesssim M_{\mathrm{pl}}$ because of the negative Hubble mass term $-c_{H} H^{2}|\phi|^{2}$. The AD field just stays there until $H=H_{\text {osc }} \simeq m_{\phi}$ because of a large damping effect, which appears in the second term in Eq. (8). The baryon number production completes as soon as the $\mathrm{AD}$ field starts the coherent oscillation around the origin at $H=H_{\text {osc }}$. In this scenario, the AD field is completely decoupled from thermal backgrounds, and the early oscillation of the $\mathrm{AD}$ field $\left(H_{\mathrm{osc}} \gg m_{\phi}\right)$ thus does not occur.

A crucial difference appearing in this scenario is the existence of a large entropy production due to decays of the $\mathrm{AD}$ field. At the reheating process of the inflation, the energy density of the inflaton is converted into the radiation. After that, the energy density of the radiation reduces as $\rho_{\text {rad }}$ $\propto R^{-4}$. On the other hand, the $\mathrm{AD}$ field starts the coherent oscillation at $H=H_{\text {osc }}$ with the initial amplitude $|\phi|=|\phi|_{0}$, and its energy density only reduces as $\rho_{\phi} \propto R^{-3}$. Then the $\mathrm{AD}$ field begins to dominate the energy density of the universe at some time, and substantial entropy is produced through decays of the AD field.

The AD field dominates the energy density of the universe before its decay if the following condition is satisfied:

$$
T_{R}>3 T_{d}\left(\frac{M_{\mathrm{pl}}}{|\phi|_{0}}\right)^{2}
$$

where $T_{d}$ is the decay temperature of the $\phi$ field, which corresponds to the decay temperature of the $Q$ ball and much lower than the weak scale, $T_{d} \ll m_{\mathrm{w}}$. In Eq. (23), we have assumed that the coherent oscillation of the AD field starts before the completion of the reheating process of the inflation. If this is not the case, i.e. if $T_{R} \geqslant 2$ $\times 10^{10} \mathrm{GeV}\left(m_{\phi} / 1 \mathrm{TeV}\right)^{1 / 2}$, the condition on $T_{d}$ becomes weaker than this case.

After the decays of the AD field, the resultant baryon asymmetry is given by the following simple form:

$$
\begin{aligned}
\frac{n_{B}}{s} & =\frac{\rho_{\phi}}{s}\left(\frac{n_{\phi}}{\rho_{\phi}}\right)\left(\frac{n_{B}}{n_{\phi}}\right)=\frac{3}{4} \frac{T_{d}}{m_{\phi}}\left(\frac{n_{B}}{n_{\phi}}\right) \\
& =7.5 \times 10^{-6}\left(\frac{T_{d}}{10 \mathrm{MeV}}\right)\left(\frac{1 \mathrm{TeV}}{m_{\phi}}\right)\left(\frac{n_{B}}{n_{\phi}}\right),
\end{aligned}
$$

where $n_{\phi}$ denotes the total number density of the $\phi$ and anti$\phi$ field. The ratio of baryon to $\phi$ number is easily estimated by an argument similar to the one given in the previous section as

$$
\left(\frac{n_{B}}{n_{\phi}}\right) \simeq|\lambda|\left(\frac{m_{3 / 2}}{m_{\phi}}\right)^{2}\left(\frac{|\phi|_{0}}{M_{\mathrm{pl}}}\right)^{2} \delta_{\mathrm{eff}},
$$

where $\delta_{\text {eff }} \equiv \sin \left[\arg (\lambda)+4 \arg \left(\phi_{0}\right)\right]$. Then a reasonable set of parameters

$$
|\lambda|\left(\frac{m_{3 / 2}}{m_{\phi}}\right)^{2}\left(\frac{|\phi|_{0}}{M_{\mathrm{pl}}}\right)^{2} \delta_{\mathrm{eff}} \sim 10^{-5}\left(\frac{10 \mathrm{MeV}}{T_{d}}\right)
$$

can naturally explain the observed baryon asymmetry.

Before the discovery of the formation of large $Q$ balls, decays of the $\mathrm{AD}$ field have been considered to occur at $T_{d}$ $\sim m_{\phi}$. If this is the case, the resultant baryon asymmetry is washed out by the sphaleron effect, since the Kähler potential adopted in the present work conserves the $B-L$ symmetry. If we adopt other interactions that violate this symmetry, ${ }^{5}$ the resultant ratio of $\phi$-number to entropy is given by $n_{\phi} / s$ $\sim \mathcal{O}(1)$, which requires a very small ratio of baryon to $\phi$-number $n_{B} / n_{\phi} \sim 10^{-10}$. Such a small ratio results in a

\footnotetext{
${ }^{5}$ Such interactions consistent with $R$-symmetry only appear at much higher orders.
} 
very unnatural fine tuning on $\lambda$ and/or $\delta_{\text {eff }}$, and hence additional large entropy productions have been considered to be necessary after the decays of the AD field, such as a thermal inflation or decays of heavy moduli fields.

Interestingly enough, the large initial amplitude of the $\mathrm{AD}$ field results in the formation of very large $Q$ balls with low decay temperature $T_{d}=\mathcal{O}(10 \mathrm{MeV}){ }^{6}$ This significantly enhances the entropy production via decays of the $\mathrm{AD}$ fields and naturally explains the observed baryon asymmetry. Higgsino or $W$-ino LSP is the necessary condition for this scenario to work, so as not to overclose the universe and explain the right amount of dark matter.

A quite beautiful point in this scenario is that the resultant baryon asymmetry and dark matter density are solely determined by the sector related to the AD field and annihilation cross section of the LSP. Any other process cannot affect the final result as long as the AD fields (stored in the $Q$ balls) dominate the energy density of the universe at their decay time. Especially, the final baryon asymmetry is completely independent of the reheating temperature of the inflation.

In addition, the so-called "cosmological gravitino problem" [31] is also solved. The dilution factor, which is the ratio of the entropy density before and after the decays of the $\mathrm{AD}$ field, is given by

$$
\frac{1}{\Delta} \equiv \frac{s_{\text {before }}}{s_{\text {after }}}=3 \frac{T_{d}}{T_{\text {ini }}}\left(\frac{M_{\mathrm{pl}}}{|\phi|_{0}}\right)^{2},
$$

where

$$
T_{\mathrm{ini}}=\min \left[T_{R}, \quad \sqrt{m_{\phi} M_{\mathrm{pl}}}\left(\frac{90}{\pi^{2} g_{*}}\right)^{1 / 4}\right],
$$

with $g_{*}$ being the effective degrees of freedom at temperature $T$. It can be seen from these relations that even if the reheating temperature of the inflation is much higher than $\sim 10^{12} \mathrm{GeV}$, the produced gravitinos are sufficiently diluted for there not to be any gravitino problem.

\section{Affleck-Dine mechanism in anomaly-mediated SUSY breaking models}

In anomaly-mediated SUSY breaking (AMSB) models, SUSY is broken in a hidden sector, and it is transmitted to the MSSM sector dominantly via the super-Weyl anomaly. The MSSM gauginos and matter fields obtain soft masses, which are one-loop-suppressed relative to the gravitino mass. Therefore, the order of the gravitino mass is estimated as $m_{3 / 2} \sim m_{\text {soft }} / \alpha \gg m_{\text {soft }}$.

A crucial point is that the $\mathrm{AD}$ mechanism always uses the nonrenormalizable operators to violate the baryon and/or lepton number, which also violate the super-Weyl symmetry at tree level. Hence, the SUSY breaking effects induced in these operators are of the order of the gravitino mass and not loop-suppressed, which generates a global minimum for the AD field displaced from the origin [32].

\footnotetext{
${ }^{6}$ See Sec. III.
}

First consider the $\mathrm{AD}$ mechanism using nonrenormalizable operators in the superpotential. In this case, the scalar potential of the $\mathrm{AD}$ field is given by Eq. (6), but with much larger gravitino mass. During the inflation, the negative Hubble mass term drives the $\mathrm{AD}$ field far from the origin and fixes it at $|\phi| \simeq\left(H_{I} M^{n-3}\right)^{1 / n-2}$. After the inflation ends, the amplitude of the AD field gradually decreases as $|\phi|$ $\simeq\left(H M^{n-3}\right)^{1 / n-2}$. At this stage, the curvature along the phase direction $m_{p h}^{2}$ is given by $m_{p h}^{2} \simeq m_{3 / 2} H$. Thus, after $H$ $\leqslant m_{3 / 2}$, this large curvature forces the AD field to settle down on the bottom of one of the valleys of the A-term potential, which are located at

$$
\arg \left(a_{m}\right)+n \arg (\phi)=\pi, \bmod \pi .
$$

Consequently, the AD field is inevitably trapped at the global minimum on the way toward the origin along the bottom of the valley:

$$
|\phi| \_\min \simeq\left(m_{3 / 2} M^{n-3}\right)^{1 /(n-2)} .
$$

This results in an $\mathrm{SU}(3)_{C}$ and/or $\mathrm{U}(1)_{\mathrm{EM}}$ breaking universe. To avoid this disaster, we need some mechanisms to stop the AD field during the inflation below "the top of the hill" in the scalar potential, which is located at

$$
|\phi|_{0} \lesssim|\phi|_{\text {hill }} \simeq\left(\frac{m_{\phi}^{2}}{m_{3 / 2}} M^{n-3}\right)^{1 /(n-2)} .
$$

The same difficulty appears in the AD mechanism making use of the nonrenormalizable operators in the Kähler potential. In this case, the potential is written as in Eq. (22), with larger gravitino mass $m_{3 / 2} \gg m_{\phi}$, and the global minimum is located near the Planck scale. In order to avoid a color and/or $\mathrm{U}(1)_{\mathrm{EM}}$ breaking universe, we need to impose the following condition:

$$
|\phi|_{0} \lesssim|\phi|_{\text {hill }}=M_{\mathrm{pl}} \frac{m_{\phi}}{m_{3 / 2}} .
$$

One natural solution is to gauge the $\mathrm{U}(1)_{B-L}$ symmetry $[24]{ }^{7}$ If the $B-L$ symmetry is gauged, flat directions along which the AD fields cannot cancel the $\mathrm{U}(1)_{B-L} D$-term contributions within themselves can be lifted at the $B-L$ breaking scale $v$ by the $D$-term potential. Then, the amplitude of the AD field can be easily suppressed by adjusting the $B$ $-L$ breaking scale as $v \leqq|\phi|_{\text {hill }}$. Even if we suppress the initial amplitude of the $\mathrm{AD}$ field, there remains plenty of room to explain the observed baryon asymmetry and to form large $Q$ balls, which allows us to obtain the right amount of $W$-ino dark matter in AMSB models.

\footnotetext{
${ }^{7}$ In Ref. [24], we make use of the gauged $B-L$ symmetry to make the $Q$ ball small enough for the produced $B$-ino LSPs not to overclose the universe. In the present work, we concentrate on the case of the Higgsino or $W$-ino LSP, so that there is no need for such an adjustment on the $B-L$ breaking scale.
} 


\section{III. $Q$ BALL}

After the flat direction field $\phi$ starts its coherent oscillation, a kind of nontopological soliton " $Q$ ball" [33] is formed because of spatial instabilities of the $\phi$ field [5-7]. In this section we briefly review the formation and the decay of the $Q$ ball.

\section{A. Size of the $Q$ ball}

The relevant scalar potential of $\phi$ at the time of $Q$-ball formation is given by

$$
V(\phi)=m_{\phi}^{2}\left[1+K \log \left(\frac{|\phi|^{2}}{M_{G}^{2}}\right)\right]|\phi|^{2},
$$

where $M_{G}$ is the renormalization scale at which the soft mass $m_{\phi}$ is defined, and the $K \log \left(|\phi|^{2}\right)$ term represents the oneloop correction. This mainly comes from the gaugino loops and $K$ is estimated in the range from -0.01 to $-0.1[6,7,23]$. Because of the potential flatter than $\phi^{2}$, spatial instabilities of the homogeneous $\phi$ field are caused after it starts the coherent oscillation [5]. In the momentum space, the instability band is given by [34]

$$
0<\frac{k^{2}}{R^{2}}<3 m_{\phi}^{2}|K|
$$

where $k$ is the comoving momentum of the fluctuations of the $\phi$ field. The best amplified mode is given by the center of the band: $\left(k^{2} / R^{2}\right)_{\max } \simeq(3 / 2) m_{\phi}^{2}|K|$. This corresponds to the radius of the $Q$ ball, which is estimated analytically using the Gaussian profile of the $Q$ ball [7]:

$$
R_{Q} \simeq \frac{1}{m_{\phi}} \sqrt{\frac{2}{|K|}}
$$

For the best amplified mode, the perturbations $\delta \phi$ of the $\phi$ field grow according to the following equation:

$$
\begin{aligned}
\left|\frac{\delta \phi}{\phi}\right| & \simeq\left|\frac{\delta \phi}{\phi}\right|_{\text {osc }} \exp \left[\int d t \frac{3 m_{\phi}|K|}{4}\right] \\
& =\left|\frac{\delta \phi}{\phi}\right|_{\text {osc }} \exp \left[\frac{m_{\phi}|K|}{2 H}\right],
\end{aligned}
$$

where we have used $H^{-1}=(3 / 2) t$, assuming that the $Q$ ball formation occurs before the reheating process of the inflation is completed. ${ }^{8}$ Therefore, the fluctuation becomes nonlinear when the Hubble parameter becomes

$$
H=H_{\mathrm{non}}=\frac{m_{\phi}|K|}{2 \alpha},
$$

where $\alpha \equiv \ln \left(|\phi|_{\text {osc }} /|\delta \phi|_{\text {osc }}\right)$ and $|\delta \phi|_{\text {osc }} \approx\left(2 \pi R_{Q}\right)^{-1}$ for the best amplified mode [35]. For the parameters we adopt in the present analyses, its typical value is given by $\alpha \simeq 30-40$. At this time $\left(H=H_{\text {non }}\right)$, the baryon number density of the AD condensation becomes

$$
n_{B}\left(t_{\text {non }}\right) \simeq n_{B}\left(t_{\mathrm{osc}}\right) \times\left(\frac{H_{\mathrm{non}}}{H_{\mathrm{osc}}}\right)^{2}
$$

Thus, from Eqs. (35), (37) and (38) and $H_{\mathrm{osc}} \simeq m_{\phi}$, the typical charge of a single $Q$ ball is estimated to

$$
\begin{aligned}
Q & \simeq \frac{4}{3} \pi R_{Q}^{3} \times n_{B}\left(t_{\text {non }}\right) \\
& \simeq \frac{2 \sqrt{2} \pi}{3} \frac{|K|^{1 / 2}}{\alpha^{2}} \frac{1}{m_{\phi}^{3}} n_{B}\left(t_{\mathrm{osc}}\right) \\
& \simeq \bar{\beta}_{a}\left(\frac{\left|\phi_{\mathrm{osc}}\right|}{m_{\phi}}\right)^{2} \epsilon,
\end{aligned}
$$

where

$$
\bar{\beta}_{a} \equiv 6 \times 10^{-4}\left(\frac{30}{\alpha}\right)^{2}\left(\frac{|K|}{0.03}\right)^{1 / 2}, \quad \epsilon \equiv \frac{n_{B}\left(t_{\mathrm{osc}}\right)}{n_{\phi}\left(t_{\mathrm{osc}}\right)}(\leqslant \beta) .
$$

Here, we have used $n_{\phi}\left(t_{\mathrm{osc}}\right) \simeq m_{\phi}|\phi|_{\text {osc }}^{2}$. This estimation of the $Q$ ball charge is roughly consistent with the result obtained by numerical lattice simulations $[34,36]$,

$$
Q \simeq \bar{\beta}_{n}\left(\frac{\left|\phi_{\mathrm{osc}}\right|}{m_{\phi}}\right)^{2} \times \begin{cases}\epsilon & \text { for } \epsilon \gtrsim \epsilon_{c} \\ \epsilon_{c} & \text { for } \epsilon \lesssim \epsilon_{c}\end{cases}
$$

where

$$
\bar{\beta}_{n} \equiv 6 \times 10^{-3}, \quad \epsilon_{c} \sim 0.01
$$

Notice that for $\epsilon \lesssim \epsilon_{c}$ the $Q$ ball charge becomes independent of the initial charge density $\epsilon$. This is because negative

\footnotetext{
${ }^{8}$ In the AD mechanism without nonrenormalizable superpotentials, the reheating temperature can be as high as $T_{R} \gtrsim 2$ $\times 10^{10} \mathrm{GeV}\left(m_{\phi} / 1 \mathrm{TeV}\right)^{1 / 2}$ and the $Q$ ball formation might occur after the reheating phase. (See Sec. II B.) In this case, $H^{-1}=2 t$, and Eq. (38) is modified as $n_{B}\left(t_{\text {non }}\right) \simeq n_{B}\left(t_{\text {osc }}\right) \times\left(H_{\text {non }} / H_{\text {osc }}\right)^{3 / 2}$. In such a case, the charge of the $Q$ ball becomes larger by a factor of $\mathcal{O}(10)$.
} 
charge $Q$ balls are created in this region [34]. In the following discussions, we shall adopt the following equation in estimating the $Q$ ball charge:

$$
Q=Q_{\max } \times\left\{\begin{array}{l}
\frac{\epsilon}{\beta} \text { for } \epsilon_{c} \lesssim \epsilon \leqslant \beta, \\
\frac{\epsilon_{c}}{\beta} \text { for } \epsilon \lesssim \epsilon_{c}
\end{array}\right.
$$

where

$$
Q_{\max } \sim 3 \times 10^{-3}\left(\frac{\left|\phi_{\text {osc }}\right|}{m_{\phi}}\right)^{2} \beta .
$$

Let us now estimate the charge of the $Q$ ball in each of the cases discussed in the previous sections. In the case of $\mathrm{AD}$ baryogenesis with a nonrenormalizable superpotential (Sec. II A), the $Q$ ball charge is estimated by using Eqs. (13) and (43), resulting in

$$
\begin{aligned}
\epsilon & \simeq \frac{2(n-2)}{3(n-3)} \beta \delta_{\text {eff }}\left|a_{m}\right|\left(\frac{m_{3 / 2}}{m_{\phi}}\right), \\
Q & \sim 3 \times 10^{-3} \times \frac{2(n-2)}{3(n-3)} \beta \delta_{\text {eff }}\left|a_{m}\right|\left(\frac{m_{3 / 2}}{m_{\phi}}\right)\left(\frac{M}{m_{\phi}}\right)^{2(n-3) /(n-2)} \\
& \simeq \begin{cases}3 \times 10^{20} \times \beta \delta_{\text {eff }}\left|a_{m}\right|\left(\frac{m_{3 / 2}}{m_{\phi}}\right)\left(\frac{1 \mathrm{TeV}}{m_{\phi}}\right)\left(\frac{M}{10^{26} \mathrm{GeV}}\right) & \text { for } n=4, \\
3 \times 10^{20} \times \beta \delta_{\text {eff }}\left|a_{m}\right|\left(\frac{m_{3 / 2}}{m_{\phi}}\right)\left(\frac{1 \mathrm{TeV}}{m_{\phi}}\right)^{3 / 2}\left(\frac{M}{M_{\mathrm{pl}}}\right)^{3 / 2} & \text { for } n=6 .\end{cases}
\end{aligned}
$$

As mentioned in Sec. II B, the $Q$ ball charge becomes larger in the AD mechanism without a nonrenormalizable superpotential, since the initial amplitude of the $\phi$ 's oscillation is large. Recall that a relatively smaller value of $\epsilon=n_{B} / n_{\phi}<\epsilon_{c}$ is preferred in order to generate the correct amount of baryon asymmetry [see Eqs. (25) and (26)]. Thus, from Eq. (43), the $Q$ ball charge is estimated to be

$$
Q \sim 10^{28}\left(\frac{|\phi|_{0}}{M_{\mathrm{pl}}}\right)^{2}\left(\frac{1 \mathrm{TeV}}{m_{\phi}}\right)^{2} \epsilon_{c},
$$

which results in $Q \sim 10^{24}-10^{26}$ for $|\phi|_{0} \simeq(0.1-1) \times M_{\mathrm{pl}}$.

Finally, in the case of the AMSB model, the initial amplitude of the AD field $\phi$ should be suppressed to avoid the unwanted trapping of the AD field at the global minimum, as discussed in Sec. II C. From Eqs. (31), (32) and (43), the $Q$ ball charge is estimated to

$$
Q \leqq 10^{-3}\left(\frac{M^{n-3}}{m_{3 / 2} m_{\phi}^{n-4}}\right)^{2 /(n-2)} \simeq \begin{cases}3 \times 10^{18} \times\left(\frac{30 \mathrm{TeV}}{m_{3 / 2}}\right)\left(\frac{M}{10^{26} \mathrm{GeV}}\right) & \text { for } n=4, \\ 2 \times 10^{19} \times\left(\frac{30 \mathrm{TeV}}{m_{3 / 2}}\right)^{1 / 2}\left(\frac{1 \mathrm{TeV}}{m_{\phi}}\right)\left(\frac{M}{M_{\mathrm{pl}}}\right)^{3 / 2} & \text { for } n=6,\end{cases}
$$

for the case with nonrenormalizable superpotentials, and

$$
Q \lesssim 10^{-3}\left(\frac{M_{\mathrm{pl}}}{m_{3 / 2}}\right)^{2} \simeq 6 \times 10^{24}\left(\frac{30 \mathrm{TeV}}{m_{3 / 2}}\right)^{2},
$$

for the case without nonrenormalizable superpotentials.

\section{B. Decay of the $Q$ ball}

Before discussing the decay of the $Q$ ball, we comment on its evaporation [37,38]. Because of the large expectation value of the scalar field inside the $Q$ ball, most of it is decoupled from the surrounding thermal bath. However, a thin outer region of the $Q$ ball is thermalized, since particles in the thermal plasma can penetrate into this region, and hence a partial evaporation of the $Q$ ball charge occurs. As stressed in Ref. [38], the evaporation of the charge from the $Q$ ball surface is suppressed by diffusion effects, and the evaporation occurs most effectively at $T \sim m_{\phi}$. The total amount of the evaporated charge is estimated to

$$
\Delta Q \sim 10^{18} \times\left(\frac{0.03}{|K|}\right)^{1 / 2}\left(\frac{1 \mathrm{TeV}}{m_{\phi}}\right),
$$

for $T_{R} \gtrsim m_{\phi}$, and

$$
\Delta Q \sim 10^{16} \times\left(\frac{0.03}{|K|}\right)^{1 / 2}\left(\frac{1 \mathrm{TeV}}{m_{\phi}}\right)^{3}\left(\frac{T_{R}}{100 \mathrm{GeV}}\right)^{2},
$$


for $T_{R} \leq m_{\phi}$. [Here, we have used $T \simeq\left(T_{R}^{2} M_{\mathrm{pl}} H\right)^{1 / 4}$ before the reheating process of the inflation is completed.] Therefore, as long as the initial charge of the $Q$ ball is larger than $\mathcal{O}\left(10^{18}\right)$, most of the $Q$-ball charge survives the evaporation. Hereafter, we assume $Q \geqslant \mathcal{O}\left(10^{18}\right) \mathrm{GeV}$ (which is naturally realized in the present scenarios, as shown in the previous sections) and discuss the emission of the remaining charge by the decay of the $Q$ ball.

The remaining charge of the $Q$ ball is emitted through its decay into light fermions. The decay rate is estimated as [39]

$$
\Gamma_{Q} \equiv-\frac{d Q}{d t} \lesssim \frac{\omega^{3} \mathcal{A}}{192 \pi^{2}},
$$

where $\mathcal{A}=4 \pi R_{Q}^{2}$ is the surface area of the $Q$ ball and $\omega$ $\simeq m_{\phi}$. This upper bound is likely to be saturated for $\phi(0)$ $\gg m_{\phi}$, where $\phi(0)$ is the field value of the AD field at the center of the $Q$ ball [39]. ${ }^{9}$ Therefore, the lifetime of a single $Q$ ball with an initial charge $Q_{i}$ is estimated to be

$$
\tau_{d}=\frac{Q_{i}}{\Gamma_{Q}} \gtrsim 1 \times 10^{-7} \sec \left(\frac{|K|}{0.03}\right)\left(\frac{1 \mathrm{TeV}}{m_{\phi}}\right)\left(\frac{Q_{i}}{10^{20}}\right),
$$

or equivalently, the decay temperature $T_{d}$ of the $Q$ ball is given by

$$
T_{d} \lesssim 2 \mathrm{GeV} \times\left(\frac{0.03}{|K|}\right)^{1 / 2}\left(\frac{m_{\phi}}{1 \mathrm{TeV}}\right)^{1 / 2}\left(\frac{10^{20}}{Q_{i}}\right)^{1 / 2} .
$$

Therefore, the $Q$ ball decays at $T_{d} \sim 1 \mathrm{MeV}$-(a few) $\mathrm{GeV}$ for the $Q$ ball charge estimated in the previous sections. It is quite interesting to observe that the $Q$ ball decay occurs just after the freeze-out of the $\operatorname{LSP}\left(T_{f} \sim m_{\chi} / 20\right)$ and just before the beginning of the big-bang nucleosynthesis (BBN) ( $T$ $\sim 1 \mathrm{MeV}$ ). Thus, the $Q$ ball decay can naturally provide both the baryon asymmetry required from the BBN and the nonthermal source of the LSP. It should also be noticed that the decay occurs after the electroweak phase transition ( $T$ $\sim 100 \mathrm{GeV})$. Thus, the produced baryon asymmetry is not washed out by the sphaleron effect, even if the $B-L$ is conserved in the $\mathrm{AD}$ mechanism, as in the $n=4$ cases and the $W=0$ cases.

\section{NONTHERMAL DARK MATTER FROM THE $Q$ BALL DECAY}

We now turn to a discussion of the nonthermal production of the LSP from the $Q$ ball decay. The relation between the $Q$ ball number density and the baryon number density is given by

$$
\begin{aligned}
& n_{B}=Q_{i}\left(n_{Q}^{+}-n_{Q}^{-}\right), \\
& n_{Q}^{\text {total }} \equiv n_{Q}^{+}+n_{Q}^{-}=\frac{n_{B}}{Q_{i}} \times\left\{\begin{array}{lll}
1 & \text { for } \quad \epsilon_{c} \lesssim \epsilon \leqslant \beta, \\
\frac{\epsilon_{c}}{\epsilon} & \text { for } \quad \epsilon \lesssim \epsilon_{c},
\end{array}\right.
\end{aligned}
$$

where $Q_{i}$ is the absolute value of the initial charge of a single $Q$ ball, which is expected to be of the same order for positive and negative charge $Q$ balls, and $n_{Q}^{+}\left(n_{Q}^{-}\right)$is the number density of positive (negative) charge $Q$ balls. Here, the negative charge $Q$ balls only appear in the case of $\epsilon \leqslant \epsilon_{c}$. Notice that almost all the baryon asymmetry is initially stored in the $Q$ balls [34].

First we assume that the $Q$ ball decay does not produce a large extra entropy. As can be seen in Eqs. (16) and (17), the AD mechanism with nonrenormalizable superpotentials can naturally provide the empirical baryon asymmetry without any extra entropy production, if the reheating temperature of the inflation is relatively low (e.g. $T_{R} \sim 100 \mathrm{GeV}$ for the $n$ $=4$ case with $M \sim 10^{26} \mathrm{GeV}$, and for the $n=6$ case with $\left.M \sim M_{\mathrm{pl}}\right)$. In these cases, the number density of the $Q$ ball is directly related to the baryon asymmetry in the present universe. It is easy to see that the energy density $\rho_{Q}$ of the $Q$ ball is much less than that of the radiation $\rho_{\text {rad }}$ for $T>T_{d}$ :

$$
\frac{\rho_{Q}}{\rho_{\mathrm{rad}}} \simeq \frac{\rho_{\phi}}{\rho_{\mathrm{rad}}}=\frac{3}{4} \epsilon^{-1} \frac{m_{\phi}}{T}\left(\frac{n_{B}}{s}\right)_{0} \lesssim 10^{-4} \times \epsilon^{-1}\left(\frac{m_{\phi}}{1 \mathrm{TeV}}\right)\left(\frac{n_{B} /\left.s\right|_{0}}{10^{-10}}\right)\left(\frac{1 \mathrm{MeV}}{T}\right) \ll 1 \quad \text { for } \quad T>T_{d} \gtrsim 1 \mathrm{MeV}
$$

Therefore, the assumption of no large extra entropy production at the $Q$ ball decay is justified for $\epsilon>10^{-4}$ $\times\left(1 \mathrm{MeV} / T_{d}\right)$. From Eq. (45), we can expect that $\epsilon$ $=\mathcal{O}(0.1)$ when we use nonrenormalizable operators in the superpotential to lift the flat direction.

The production rate of the LSP per unit time per unit volume is given by

\footnotetext{
${ }^{9}$ If kinematically allowed, there are also decay channels into lighter scalars through 3-point couplings. Even if this is the case, the decay rate is at most comparable to that in Eq. (52).
}

$$
\begin{aligned}
\left(\frac{d n_{\chi}}{d t}\right)_{\text {prod }} & =N_{\chi} \Gamma_{Q} n_{Q}^{\text {total }}=N_{\chi} s\left(\frac{n_{B}}{s}\right)_{0} \frac{\theta\left(\tau_{d}-t\right)}{\tau_{d}} \times f(\epsilon) \\
f(\epsilon) & \equiv\left\{\begin{array}{lll}
1 & \text { for } \quad \epsilon_{c} \lesssim \epsilon \leqslant \beta, \\
\frac{\epsilon_{c}}{\epsilon} & \text { for } & \epsilon \lesssim \epsilon_{c},
\end{array}\right.
\end{aligned}
$$

where $N_{\chi}$ is the number of LSPs produced per baryon number, which is at least $N_{\chi}^{\mathrm{min}}=[\epsilon f(\epsilon)]^{-1} \geqslant 3$. 
The evolution of the number density of the LSP is then described by the following Boltzmann equation:

$$
\dot{n}_{\chi}+3 H n_{\chi}=N_{\chi} s\left(\frac{n_{B}}{s}\right)_{0} \frac{\theta\left(\tau_{d}-t\right)}{\tau_{d}} f(\epsilon)-\langle\sigma v\rangle n_{\chi}^{2},
$$

where $\langle\sigma v\rangle$ is the thermally averaged annihilation cross section of the LSP. ${ }^{10}$ Here, we have neglected the effect of the pair production of the LSPs, which is suppressed by a Boltzmann factor $\exp \left(-m_{\chi} / T\right)$ for $T<m_{\chi}$.

If the $Q$ ball decay produces significant entropy, such as the AD mechanism without a superpotential as discussed in Sec. II B, $n_{Q}$ is not directly related to the present baryon asymmetry. In this case, the evolution of the number density of the LSPs $n_{\chi}$ is governed by the following coupled equations:

$$
\dot{n}_{\chi}+3 H n_{\chi}=N_{\chi} \Gamma_{Q} n_{Q}^{\text {total }}-\langle\sigma v\rangle n_{\chi}^{2},
$$

where $n_{Q}^{\text {total }}$ is given by

$$
\begin{aligned}
& \dot{n}_{Q}^{\text {total }}+3 H n_{Q}^{\text {total }}=0\left(\text { for } \quad t \leqslant \tau_{d}\right), \\
& n_{Q}^{\text {total }}=0 \quad\left(\text { for } \quad t>\tau_{d}\right),
\end{aligned}
$$

and the Hubble parameter $H$ is obtained from the Friedmann equation:

$$
H^{2}=\frac{1}{3 M_{\mathrm{pl}}^{2}}\left(\rho_{Q}+\rho_{\chi}+\rho_{\mathrm{rad}}\right)
$$

where

$$
\begin{aligned}
\rho_{Q}=\rho_{\phi}= & {[\epsilon f(\epsilon)]^{-1} m_{\phi}\left(Q_{i}-\Gamma_{Q} t\right) n_{Q}^{\text {total }}, } \\
\rho_{\chi}= & m_{\chi} n_{\chi} \\
\dot{\rho}_{\mathrm{rad}}+4 H \rho_{\mathrm{rad}}= & \left\{[\epsilon f(\epsilon)]^{-1} m_{\phi}-N_{\chi} m_{\chi}\right\} \Gamma_{Q} n_{Q}^{\text {total }} \\
& +m_{\chi}\langle\sigma v\rangle n_{\chi}^{2} .
\end{aligned}
$$

Although the Boltzmann equations have complicated forms, the final abundance of the LSPs can be approximately expressed by a simple analytical form. Note that the Boltzmann equations for the LSP [Eqs. (58) and (59)] are reduced to the following one for $t>\tau_{d}$ :

$$
\dot{n}_{\chi}+3 H n_{\chi}=-\langle\sigma v\rangle n_{\chi}^{2} \quad\left(\text { for } \quad t>\tau_{d}\right) .
$$

It may be useful to rewrite this equation in terms of $Y_{\chi}$ $=n_{\chi} / s$ and the temperature $T$ :

$$
\frac{d Y_{\chi}}{d T}=\sqrt{\frac{8 \pi^{2} g_{*}}{45}}\left(1+\frac{T}{g_{*}} \frac{d g_{*}}{d T}\right)\langle\sigma v\rangle M_{\mathrm{pl}} Y_{\chi}^{2} .
$$

\footnotetext{
${ }^{10}$ The LSPs are likely to be in kinetic equilibrium at least for $T$ $\gtrsim \mathcal{O}(\mathrm{MeV})[40]$.
}

We assume that the $s$-wave contributions dominate the annihilation cross section of the neutralino, which is a reasonable approximation for $\widetilde{H}$ - and $\widetilde{W}$-like LSP with $T_{d} \ll m_{\chi}$. By using the approximations $g_{*}(T) \simeq g_{*}\left(T_{d}\right) \simeq$ const and $\langle\sigma v\rangle(T) \simeq$ const, it can be solved analytically:

$$
Y_{\chi}(T) \simeq\left[\frac{1}{Y_{\chi}\left(T_{d}\right)}+\sqrt{\frac{8 \pi^{2} g_{*}\left(T_{d}\right)}{45}}\langle\sigma v\rangle M_{\mathrm{pl}}\left(T_{d}-T\right)\right]^{-1} .
$$

If the initial abundance $Y_{\chi}\left(T_{d}\right)$ is large enough, the final abundance $Y_{\chi 0}$ for $T \ll T_{d}$ is given by

$$
Y_{\chi 0} \simeq Y_{\chi}^{\mathrm{approx}} \equiv\left[\sqrt{\frac{8 \pi^{2} g_{*}\left(T_{d}\right)}{45}}\langle\sigma v\rangle M_{\mathrm{pl}} T_{d}\right]^{-1} .
$$

Therefore, in this case, the final abundance $Y_{\chi^{0}}$ is determined only by the $Q$ ball decay temperature $T_{d}$ and the annihilation cross section of the LSP $\langle\sigma v\rangle$, independently of the initial value $Y_{\chi}\left(T_{d}\right)$ as long as $Y_{\chi}\left(T_{d}\right) \gg Y_{\chi}^{\text {approx }}$. In terms of the density parameter $\Omega_{\chi}$, it is rewritten as

$$
\begin{aligned}
\Omega_{\chi} & =0.5\left(\frac{0.7}{h}\right)^{2}\left(\frac{m_{\chi}}{100 \mathrm{GeV}}\right)\left(\frac{10^{-7} \mathrm{GeV}^{2}}{\langle\sigma v\rangle}\right) \\
& \times\left(\frac{100 \mathrm{MeV}}{T_{d}}\right)\left(\frac{10}{g_{*}\left(T_{d}\right)}\right)^{1 / 2},
\end{aligned}
$$

where $h$ is the present Hubble parameter in units of $100 \mathrm{~km} \mathrm{sec}^{-1} \mathrm{Mpc}^{-1}$ and $\Omega_{\chi} \equiv \rho_{\chi} / \rho_{c}$. ( $\rho_{\chi}$ and $\rho_{c}$ are the energy density of the LSP and the critical energy density in the present universe, respectively.)

In the case of $Y_{\chi}\left(T_{d}\right)<Y_{\chi}^{\text {approx }}$, the final abundance is given by

$$
Y_{\chi 0} \simeq Y_{\chi}\left(T_{d}\right) \geq \epsilon^{-1}\left(\frac{n_{B}}{s}\right)_{0} .
$$

This is the case for the LSP whose annihilation cross section is small enough, such as the $B$-ino-like LSP. In this case, there is a very interesting feature that the relic abundance of the LSPs is directly related to the observed baryon asymmetry [7]. Unfortunately, however, the relics of the $B$-ino-like neutralino overclose the universe unless we assume an extremely light $B$-ino. This is easily seen from the following density parameter:

$$
\Omega_{\chi} \geqslant \epsilon^{-1}\left(\frac{m_{\chi}}{m_{p}}\right) \Omega_{B},
$$

where $m_{p}$ is the mass of the nucleon. Therefore, the $B$-ino mass should be lighter than

$$
m_{\chi} \leqslant 1 \quad \mathrm{GeV} \times\left(\frac{\epsilon}{0.1}\right)\left(\frac{\Omega_{\chi}}{10 \Omega_{B}}\right) .
$$

We have numerically solved the Boltzmann equations for the case without a large entropy production [Eq. (58)] and for the case with a large entropy production [Eqs. (59)-(64)]. 


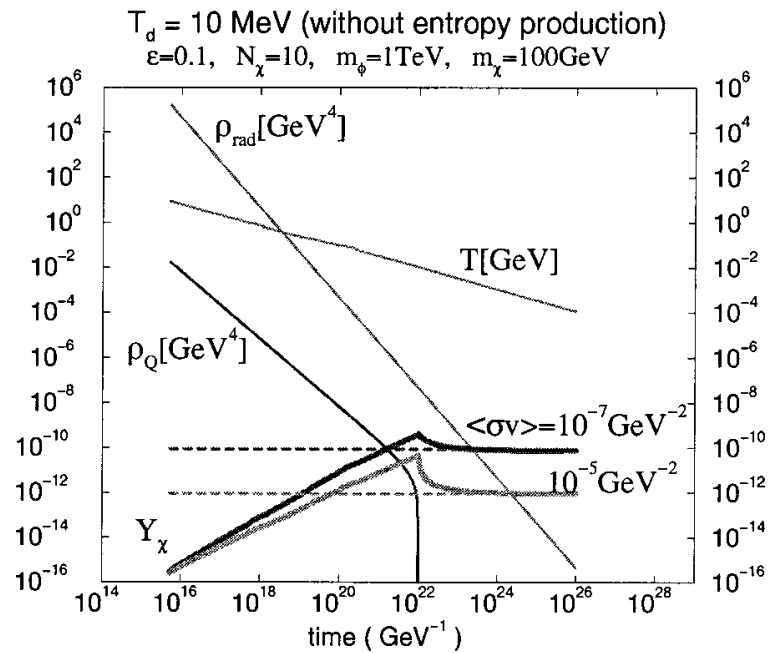

FIG. 1. The evolution of the abundance of the neutralino dark matter generated from the $Q$ ball decay for $T_{d}=10 \mathrm{MeV}$ with $\langle\sigma v\rangle=10^{-7} \mathrm{GeV}^{-2}$ and $10^{-5} \mathrm{GeV}^{-2}$, which are represented by thick solid lines. The abundances estimated by the analytic formula in Eq. (68) are shown in dashed lines. In this figure, we have assumed that the energy density of the $Q$ ball is small enough with respect to that of the radiation. The parameters are taken to be $m_{\phi}$ $=1 \mathrm{TeV}, m_{\chi}=100 \mathrm{GeV}, \epsilon=0.1$ and $N_{\chi}=10$.

Here, we have used the following set of parameters: $N_{\chi}$ $=10, \epsilon=0.1,\left(n_{B} / s\right)_{0}=0.7 \times 10^{-10}, m_{\phi}=1 \mathrm{TeV}$ and $m_{\chi}$ $=100 \mathrm{GeV}$ for the former case, and $N_{\chi}=100, \epsilon<\epsilon_{c}$ $=0.01, m_{\phi}=1 \mathrm{TeV}$ and $m_{\chi}=100 \mathrm{GeV}$ for the latter case. As can be seen from Figs. 1-4, the numerical calculations reproduce the simple analytic estimation in Eq. (68) quite well. Therefore, we use the analytic estimation in Eqs. (68) and (69) for the relic abundance of the neutralinos in the remainder of this paper, since it gives us the correct relic abundance of the LSPs as long as it is the required mass density as dark matter.

Before closing this section, we should comment on the distribution of the LSPs. So far, we have assumed that they

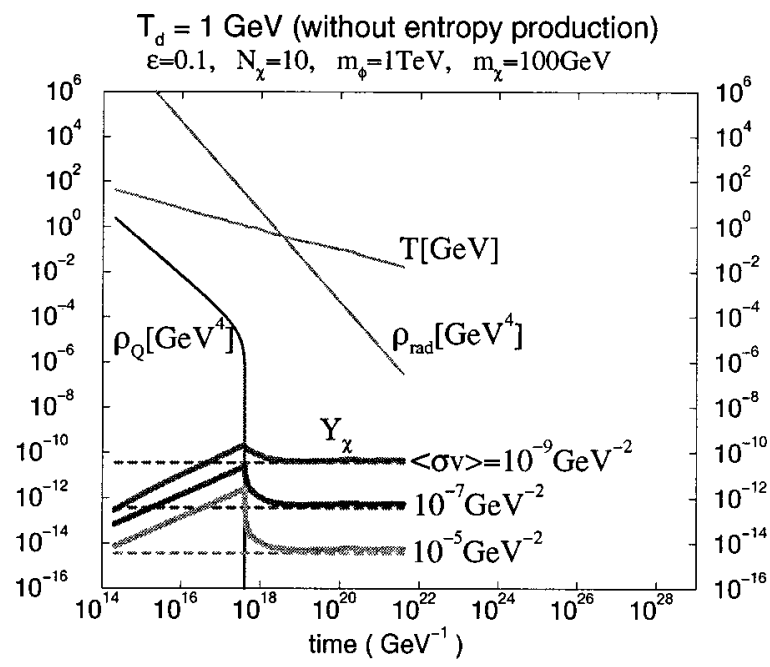

FIG. 2. The same as Fig. 1, but with $T_{d}=1 \mathrm{GeV}$ and $\langle\sigma v\rangle$ $=10^{-9}, 10^{-7}$, and $10^{-5} \mathrm{GeV}^{-2}$.

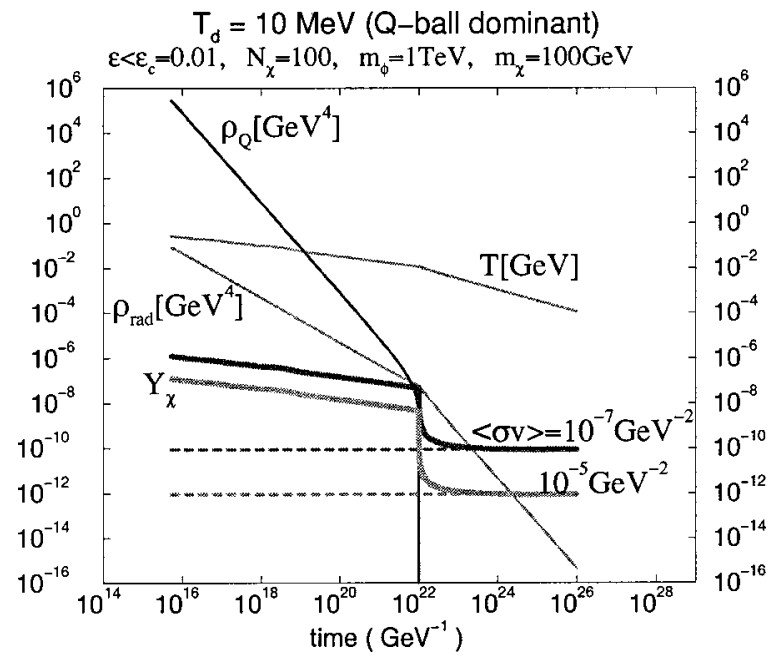

FIG. 3. The evolution of the abundance of the neutralino dark matter generated from the $Q$ ball decay for $T_{d}=10 \mathrm{MeV}$ with $\langle\sigma v\rangle=10^{-7} \mathrm{GeV}^{-2}$ and $10^{-5} \mathrm{GeV}^{-2}$, which are represented by thick solid lines. The abundances estimated by the analytic formula in Eq. (68) are shown in dashed lines. In this figure, we have assumed that the $Q$ balls dominate the energy density of the universe before their decay. The parameters are taken to be $m_{\phi}=1 \mathrm{TeV}$, $m_{\chi}=100 \mathrm{GeV}, \epsilon<\epsilon_{c}=0.01$ and $N_{\chi}=100$.

are uniformly distributed after they are produced by the $Q$ ball decay. One might wonder if the LSPs are localized near the $Q$ ball, since the $Q$ ball is a localized object. If this is the case, the pair annihilation rate of the LSP is enhanced and the final abundance might become smaller. Here, we show that this is not the case. As can be seen in Eq. (67), the abundance of LSPs approaches its final value only after $\left(T_{d}-T\right) / T_{d} \simeq \mathcal{O}(1)$, which means it takes a time scale $\Delta t$ $\sim \tau_{d}$. (This is independent of the local abundance, as long as it is large enough.) By that time, LSPs have spread out from the decaying $Q$ ball by a random walk colliding with background particles. Then the LSPs produced from a single $Q$ ball form a Gaussian distribution around that $Q$ ball, whose

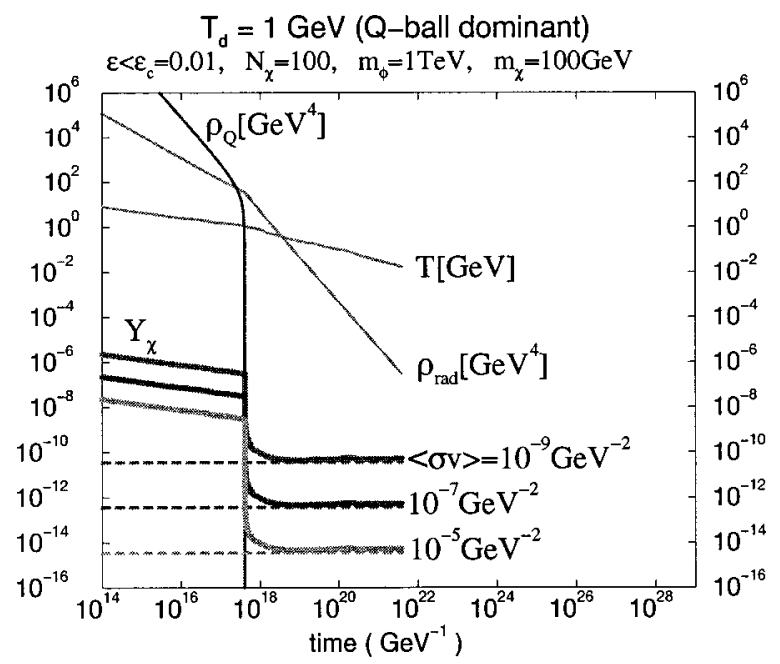

FIG. 4. The same as Fig. 3, but with $T_{d}=1 \mathrm{GeV}$ and $\langle\sigma v\rangle$ $=10^{-9}, 10^{-7}$, and $10^{-5} \mathrm{GeV}^{-2}$. 
radius is given by $\bar{r} \simeq \sqrt{\nu \tau_{d}}$, where $\nu^{-1} \simeq G_{F}^{2} m_{\chi} T_{d}^{4}$ [7]. $\left(G_{F}\right.$ is the Fermi constant.) The number of the $Q$ balls within this radius is given by $(4 \pi / 3) \bar{r}^{3} n_{Q} \sim 10^{10}$ $\times\left(T_{d} / 1 \mathrm{GeV}\right)^{-6}\left(Q_{i} / 10^{20}\right)^{-1}\left(m_{\chi} / 100 \mathrm{GeV}\right)^{-3 / 2}$, which is much larger than 1 . Therefore, the assumption of an uniform distribution is justified.

\section{DIRECT AND INDIRECT DETECTIONS OF HIGGSINO AND $W$-INO NONTHERMAL DARK MATTER FROM AFFLECK-DINE BARYOGENESIS}

In this section, we investigate the prospects of direct and indirect detection of nonthermal dark matter resulting from the late-time decays of $Q$ balls in several SUSY breaking models. The requirement that the LSPs produced via the latetime decays of $Q$ balls do not overclose the universe leads to the parameter region where the annihilation cross section of the LSP is substantially large. The possible LSP candidates are Higgsino $\widetilde{H}$ and $W$-ino $\widetilde{W} .^{11}$

The most promising way to confirm the existence of neutralino dark matter is given by a direct detection through elastic scatterings of neutralinos with matter. The interactions of neutralinos with matter are usually dominated by scalar couplings for relatively heavy nuclei $A \gtrsim 20$ [41,42]. These interactions are mediated by the light $h$ and heavy $H$ Higgs exchanges, or an sfermion $\tilde{f}$ exchange. Notice that the former interactions contain $h \chi \chi$ and $H \chi \chi$ couplings, which are suppressed for $B$-ino-like LSPs. In the case of the $W$-inolike dark matter, these couplings are enhanced by the factor $g 2 /\left(g 1 \tan \theta_{W}\right)$. As for the Higgsino-like dark matter (except for the case of the pure Higgsino LSP), these couplings are highly enhanced by the mixing with the gaugino components in the LSP. As we will see, these effects give us an intriguing possibility to detect the nonthermal dark matter in next generation direct dark matter searches, such as CDMS [43], CRESST [44], EDELWEISS II [45], GENIUS [46] and ZEPLIN MAX [47].

Many other indirect detection methods open up if a significant portion of halo dark matter consists of the neutralino LSP. These indirect methods utilize the fact that neutralinos accumulated in the halo or in massive bodies such as the Earth or the Sun may annihilate, resulting in ordinary particles, which can be detected. For example, fluxes of antiprotons and positrons, which are not produced in large quantities by cosmic rays, can be enhanced by the annihilation of neutralinos accumulated in the halo. Although the fluxes could be a measurable size, a clear discrimination from the background is difficult, because of the rather featureless spatial and energy distributions of these antiparticles. A much better signature is provided by neutralino annihilation into neutri-

\footnotetext{
${ }^{11}$ Here, we do not mean the pure Higgsino and $W$-ino LSP. A significant fraction of the $B$-ino component is possible. In fact, this is the case for most of the parameter regions in the MSUGRA scenario we will present in this section. In the case of large $\tan \beta$, even the $B$-ino-like LSP is possible to achieve the desired mass density of dark matter.
}

nos near the center of the Sun or the Earth. Since the ordinary solar neutrinos only have energies at most of the order of $\mathrm{MeV}$, a multi-GeV neutrino signal from the Sun and the Earth may give us unmistakable evidence, although there are always isotropic and anisotropic backgrounds coming from neutrinos created on the other side of the Earth and in the outer region of the Sun by cosmic rays, respectively.

In the present work, we investigate another promising way of detecting neutralinos in the halo. An excellent signature is provided by neutralino annihilation into the $2 \gamma$ final state through one-loop diagrams [11]. ${ }^{12}$ This annihilation channel leads to monoenergetic $\gamma$-rays with energy $\simeq m_{\chi}$. Although there is an extragalactic $\gamma$ ray background and backgrounds of gamma-like hadronic and electron showers, the signal will stand out against them in favorable circumstances. In fact, this is the case for the present scenario. As we will see, a large fraction of the $\widetilde{H}$ or $\widetilde{W}$ component in the LSP required from the late-time $Q$ ball decays significantly enhances the annihilation cross section via diagrams including a chargino- $W$ boson loop, which gives us an intriguing possibility to observe the $\gamma$-ray lines in the next generation of air Cherenkov telescopes observing the galactic center, such as VERITAS [49], HESS [50] and MAGIC [51].

In this section, we specify the parameter region where the neutralino LSPs, produced nonthermally through late-time decays of $Q$ balls, give a desired mass density of dark matter. In the estimation of the annihilation cross section of the neutralino, we have used all the tree-level annihilation channels with nonzero $s$-wave contributions $[52,42]$. Here, we have neglected the possible co-annihilation effects with the lightest chargino, which is justified as long as the decay temperature of $Q$ balls is lower than the mass difference between the LSP and the lightest chargino. As we will see, this condition is satisfied in most of the parameter region. Anyway, we are not interested in the difference by a factor of $\mathcal{O}(1)$ in the estimation of the relic abundance, since there are ambiguities in the estimation of the decay temperature of $Q$ balls.

We also perform calculations of the neutralino-proton scalar cross section $\sigma_{P}$ and annihilation cross section into the $2 \gamma$ final states $\sigma_{2 \gamma}$ in several SUSY breaking models: these include the "focus point" [10] in a MSUGRA scenario, the anomaly-mediated SUSY breaking model [8] with additional universal soft scalar masses, and the no-scale model with nonuniversal gaugino masses [9]. Finally, we comment on the possibility that the nonthermally produced LSPs via $Q$-ball decays form the warm dark matter.

\section{A. Parameter space and possibility of detection in a MSUGRA scenario}

In the framework of minimal supergravity (MSUGRA), there are four continuous free parameters and one binary choice:

\footnotetext{
${ }^{12}$ Neutralino annihilation into the $Z \gamma$ final state has a similar signature. For a detailed discussion of the indirect detections utilizing the neutralino annihilation channel including a photon final state, see Ref. [48].
} 


$$
m_{0}, M_{1 / 2}, A_{0}, \tan \beta, \operatorname{sgn}(\mu),
$$

where $m_{0}, M_{1 / 2}, A_{0}$ are the universal soft scalar mass, gaugino mass, and trilinear scalar coupling given at the grand unified theory (GUT) scale $M_{G} \simeq 2 \times 10^{16} \mathrm{GeV}$, respectively. All the couplings and mass parameters at the weak scale are obtained through the renormalization group (RG) evolution. In our work, we use the SOFTSUSY code [53] to calculate quantities at the weak scale, which include twoloop RG equations, one-loop self-energies for all the particles and one-loop threshold corrections from SUSY particles to the gauge and Yukawa coupling constants following the methods of Ref. [54].

At the weak scale, the Higgsino mass parameter $|\mu|$ is determined by the condition of electroweak symmetry breaking, which, at tree level, is given by

$$
\frac{1}{2} m_{Z}^{2}=\frac{m_{H_{d}}^{2}-m_{H_{u}}^{2} \tan ^{2} \beta}{\tan ^{2} \beta-1}-\mu^{2} \simeq-m_{H_{u}}^{2}-\mu^{2},
$$

where the last relation holds for moderate and large $\tan \beta$ $\geq 5$.

Unfortunately, in a MSUGRA scenario, the $B$-ino-like LSP is realized in most of the parameter space where even the thermal relics of the $B$-ino LSPs overclose the universe. In such a region, of course, the $B$-ino LSPs nonthermally produced through decays of $Q$ balls only make the problem worse.

However, there exists an interesting region where the $\widetilde{H}$-like LSP is naturally realized. The crucial observation is the existence of a "focus point" behavior in the weak scale value of the soft scalar mass of the up-type Higgs multiplet, $m_{H_{u}}^{2}$ [10]. The weak scale value of $m_{H_{u}}^{2}$ remains the weak scale even for multi-TeV $m_{0}$, as long as $M_{1 / 2}$ and $A_{0}$ are set to be around the weak scale. Therefore, from the relation in Eq. (74), one can see that the electroweak scale is insensitive to the input parameters for relatively large $\tan \beta$. By virtue of this focus point behavior, multi-TeV values of $m_{0}$ do not require strong fine tunings on the input parameters and are natural in that sense. Such large values of $m_{0}$ give positive contributions to $m_{H_{u}}^{2}$, making it less negative. This, in turn, leads to smaller values of $|\mu|$ at the weak scale. Therefore, as $m_{0}$ increases further, the $\widetilde{H}$ content in the lightest neutralino $\chi$ increases, until it finally enters the region $|\mu|$ $\lesssim 105 \mathrm{GeV}$, which is excluded by chargino searches at the CERN $e^{+} e^{-}$collider LEP II [55].

In the focus point region, the large $\widetilde{H}$ content of the LSP enhances the neutralino annihilation cross section into the $W$ bosons via chargino exchange, which is not helicitysuppressed. This naturally results in a desired mass density of dark matter via the late-time decays of $Q$ balls.

In Fig. 5, we show the allowed region for $\tan \beta=15$ in the $\left(m_{0}-M_{1 / 2}\right)$ plane. Here we take the sign of $\mu$ to be positive and $A_{0}=0$. As for the criterion to select the region, we have imposed the following conditions:

$$
0.05 \leqslant \Omega_{\chi} h^{2} \leqslant 0.5
$$

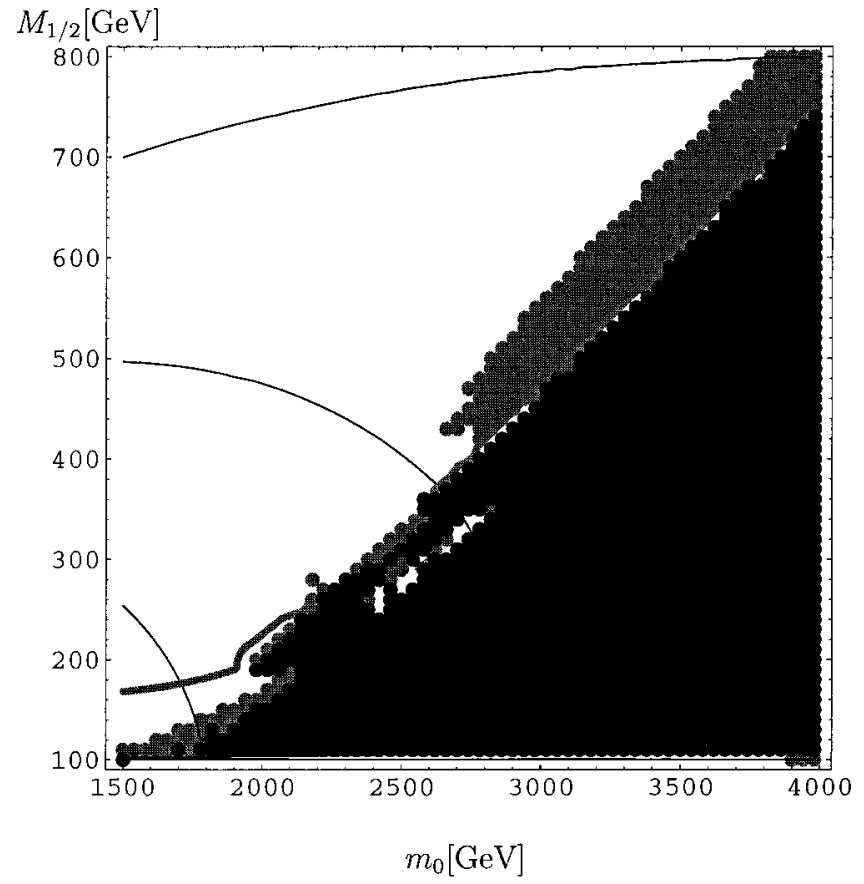

FIG. 5. The allowed region in the MSUGRA scenario with $\tan \beta=15$ and $A_{0}=0$ in the $\left(m_{0}-M_{1 / 2}\right)$ plane. In the dark shaded region, nonthermally produced LSPs via decays of $Q$ balls result in a cosmologically interesting mass density. The black shaded region is where the electroweak symmetry breaking cannot be implemented. The region below the thick line is excluded by the chargino mass bound $m_{\chi^{ \pm}} \gtrsim 105 \mathrm{GeV}$. The contours of the light Higgs boson mass are given by the thin lines, which correspond to $m_{h}$ $=117,120,122 \mathrm{GeV}$, respectively. There is no region excluded by the bounds on the $b \rightarrow s \gamma$ branching ratio.

$$
1 \mathrm{MeV} \leqslant T_{d} \leqslant 10 \mathrm{GeV} .
$$

We will use these conditions throughout this paper.

In the dark shaded region, the nonthermally produced LSPs result in a cosmologically interesting mass density. In the allowed region, the content of the LSP is dominated by $\widetilde{H}$. A typical decay temperature of $Q$ balls, which leads to the desired mass density of dark matter, is $100 \mathrm{MeV} \lesssim T_{d}$ $\lesssim$ (a few) GeV. On the other hand, we have confirmed that the mass difference between the $\widetilde{H}$-like LSP and the lightest chargino is at least $\mathcal{O}(10) \mathrm{GeV}$. Hence, the co-annihilation effects are safely neglected. This is also true for the case of $\tan \beta=40$, which will be discussed later. The electroweak symmetry breaking does not occur in the black shaded region. The region below the thick line is excluded by the chargino mass limit, $m_{\chi^{ \pm}} \gtrsim 105 \mathrm{GeV}$. The thin lines are the contours of the light Higgs boson mass, which are 117,120 and $122 \mathrm{GeV}$, respectively. We also calculate the branching ratio of the $b \rightarrow s \gamma$ transition [56]. We adopt the following constraints on the $b \rightarrow s \gamma$ branching ratio obtained by the CLEO experiment [57]:

$$
2 \times 10^{-4}<B\left(B \rightarrow X_{s} \gamma\right)<4 \times 10^{-4} .
$$

In Fig. 5, there is no region excluded by these bounds. 


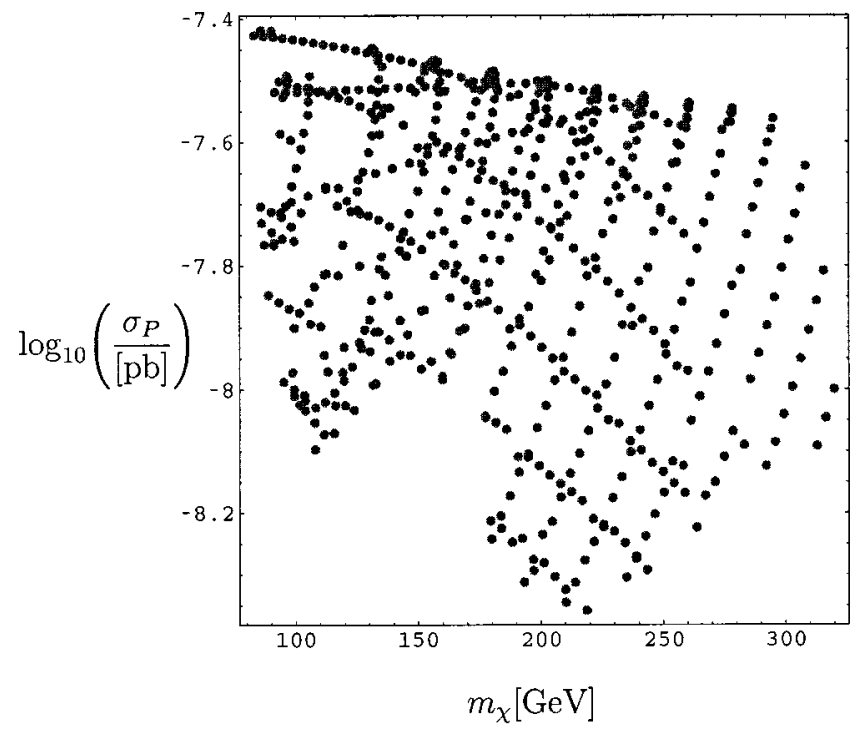

FIG. 6. Proton- $\chi$ cross section in the MSUGRA scenario for the dark shaded region in Fig. 5, also with the chargino mass bound.

In general, the matter- $\chi$ cross sections are dominated by spin-independent couplings for relatively heavy nuclei, $A$ $\gtrsim 20$. Assuming this is the case, we can obtain the detection rate of the neutralinos for each detector material by scaling the proton- $\chi$ cross section. In Fig. 6, we show this cross section in $\mathrm{pb}$ units in a MSUGRA scenario with $\tan \beta=15$. Each point in this scattered plot corresponds to one parameter set in the dark shaded region above the chargino mass bound in Fig. 5. In this calculation, we adopt the following values of the proton matrix elements for each of the three light quarks:

$$
f_{T u}=0.019, f_{T d}=0.041, f_{T s}=0.14,
$$

where $f_{T q} \equiv\left\langle p\left|m_{q} \bar{q} q\right| p\right\rangle / m_{p}$. For details about the calculation of the proton $-\chi$ cross section, see Refs. [58,42]. One can see from the figure that the proton- $\chi$ cross section satisfies $\sigma_{P} \gtrsim 10^{-8} \mathrm{pb}$ in most of the parameter space, which is within the reach of various next-generation detectors [4347].

Finally, we show the annihilation rate of neutralinos into the $2 \gamma$ final states in Fig. 7. Here we use the result of a full one-loop calculation presented in Ref. [11]. The large $\widetilde{H}$ component of the neutralino enhances the annihilation rate, which even reaches $2 v \sigma_{2 \gamma} \sim 10^{-28} \mathrm{~cm}^{3} \mathrm{sec}^{-1}$ in regions where the LSP is almost pure $\widetilde{H}$. The fact that the $\widetilde{H}$-like neutralino gives such a large annihilation rate was already known and investigated in Refs. [11,59]. However, in the previous works, the thermal relic of neutralinos was assumed to provide an appropriate mass density of dark matter. Therefore, the $\widetilde{H}$-like neutralino only appears in the region with very large mass, $m_{\chi} \gtrsim 500 \mathrm{GeV}$, because of a large annihilation cross section. In contrast, the late-time decays of $Q$ balls lead to relatively light neutralino dark matter with a significant $\widetilde{H}$ component. This is much more preferable regarding naturalness as well as detection.

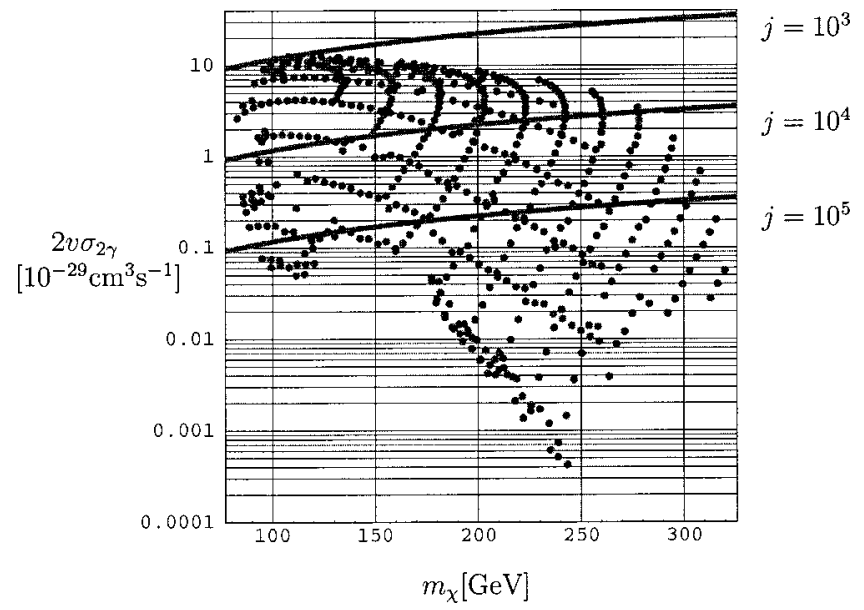

FIG. 7. Annihilation rate of neutralinos into the $2 \gamma$ final state in the MSUGRA scenario for the dark shaded region in Fig. 5, also with the chargino mass bound.

The $\gamma$-ray flux in a given direction of the sky is obtained by integrating the contributions along the line of sight (l.o.s.). The result highly depends on models of the density profile of the halo. Fortunately, all the model dependences can be factored out in terms of the following dimensionless function:

$$
J(\psi)=\frac{1}{8.5 \mathrm{kpc}} \cdot\left(\frac{1}{0.3 \mathrm{GeV} \mathrm{cm}^{-3}}\right)^{2} \int_{\text {l.o.s. }} \rho^{2}(l) d l(\psi),
$$

where $\psi$ is the angle between the direction of the galactic center and that of observation; $\rho(l)$ denotes the density profile of the halo along the l.o.s.. The $\gamma$-ray flux is written by this function, the neutralino mass and the cross section:

$$
\begin{aligned}
\Phi_{\gamma}(\psi) \simeq & 1.87 \times 10^{-11}\left(\frac{N_{\gamma} v \sigma}{10^{-29} \mathrm{~cm}^{3} \mathrm{sec}^{-1}}\right)\left(\frac{10 \mathrm{GeV}}{m_{\chi}}\right)^{2} \\
& \times J(\psi) \mathrm{cm}^{-2} \mathrm{sec}^{-1} \mathrm{sr}^{-1}
\end{aligned}
$$

where $N_{\gamma}$ is the number of photons in the final state, and $N_{\gamma}=2$ in the $\chi \chi \rightarrow \gamma \gamma$ annihilation.

Although the maximum flux will be obtained in the direction of the galactic center $\psi=0$, the experimentally relevant value is the integral of $J(\psi)$ over the solid angle around $\psi$ $=0$ determined by the angular acceptance of a detector. Therefore, the relevant function we have to treat is

$$
\langle J(0)\rangle(\Delta \Omega)=\frac{1}{\Delta \Omega} \int_{\Delta \Omega} J(\psi) d \Omega,
$$

where $\Delta \Omega$ is the angular acceptance of the detector. The value of $\langle J(0)\rangle$ and its dependence on $\Delta \Omega$ in various halo models are discussed in Ref. [59]. A typical value is $\Delta \Omega$ $=10^{-3} \mathrm{sr}$ for a generic next-generation air Cherenkov telescope (ACT). We define the value of $\langle J(0)\rangle$ averaged over this angular acceptance as $j=\langle J(0)\rangle\left(10^{-3} \mathrm{sr}\right)$. 


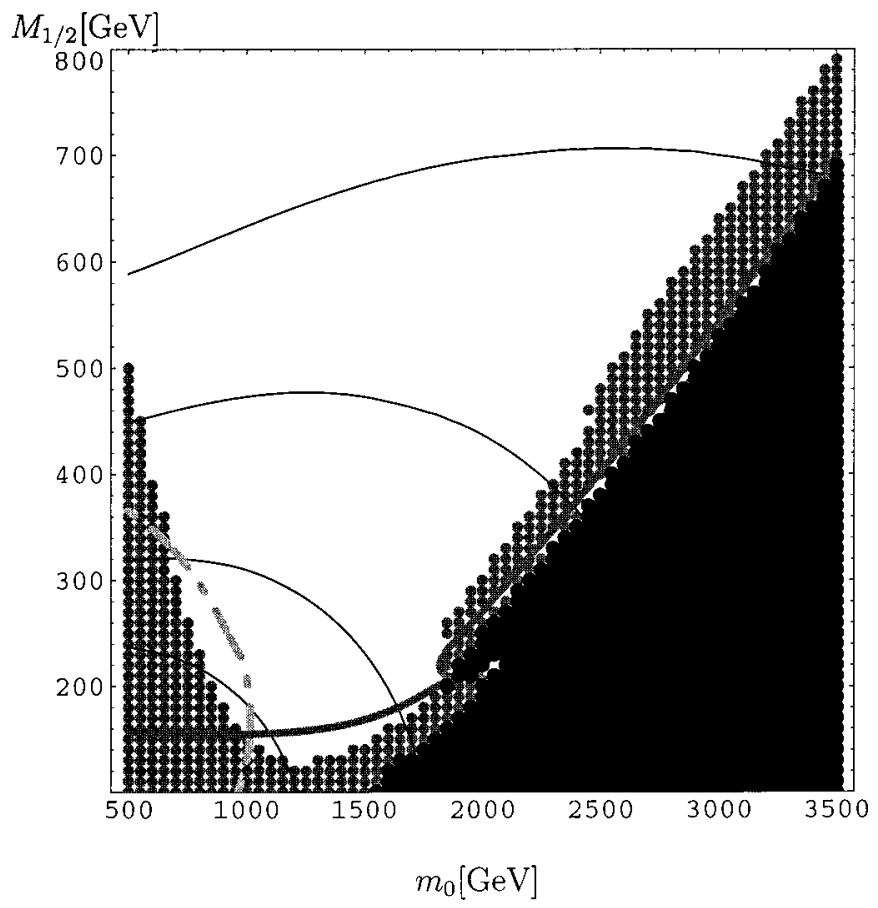

FIG. 8. The allowed region in the MSUGRA scenario with $\tan \beta=40$ and $A_{0}=0$ in the $\left(m_{0}-M_{1 / 2}\right)$ plane. In the dark shaded region, nonthermally produced LSPs via decays of $Q$ balls result in a cosmologically interesting mass density. The black shaded region is where the electroweak symmetry breaking cannot be implemented. The region below the thick line is excluded by the chargino mass bound $m_{\chi^{ \pm}} \gtrsim 105 \mathrm{GeV}$. The contours of the light Higgs boson mass are given by the thin lines, which correspond to $m_{h}$ $=114.1,117,120,122 \mathrm{GeV}$, respectively. The region below the dotdashed line is excluded where the branching ratio of $b \rightarrow s \gamma$ violates the CLEO bound, $B\left(B \rightarrow X_{s} \gamma\right)>2 \times 10^{-4}$.

In Fig. 7, we also plot $5 \sigma$ sensitivity curves for typical next generation ACT arrays [49-51] observing the galactic center, adopting several models of dark matter distribution, including a Moore et al. profile [60] for $j=10^{5}$ and a Navarro, Frenk and White profile [61] for $j=10^{3}$. In obtaining the sensitivities curves, we consider an instrument with a $10^{9} \mathrm{~cm}^{2}$ effective area and $15 \%$ energy resolution, assuming $10^{6} \mathrm{sec}$ exposure and using the standard estimation of the background presented in Ref. [59]. We have also adopted a hadronic rejection that is improved by a factor of 16 with respect to the current Whipple detector, following the arguments presented in this reference. One can see that by assuming somewhat cuspy dark matter profiles of the halo, we have a relatively large possibility for detecting monoenergetic $\gamma$-rays, especially for the region with almost pure Higgsino LSP.

In Figs. 8, 9 and 10, we show, respectively, the allowed parameter space, the proton- $\chi$ cross sections and $\chi$-annihilation rates into $2 \gamma$ final state for the MSUGRA scenario with $\tan \beta=40$. We take $\operatorname{sgn}(\mu)$ to be positive also in this case, which is desirable to avoid a large branching ratio of the $b \rightarrow s \gamma$ transition. Conventions are the same as in Figs. 5, 6 and 7. One can see some differences in Fig. 8 compared with Fig. 5. First, the region below the dot-dashed line is excluded by the constraints on the branching ratio of

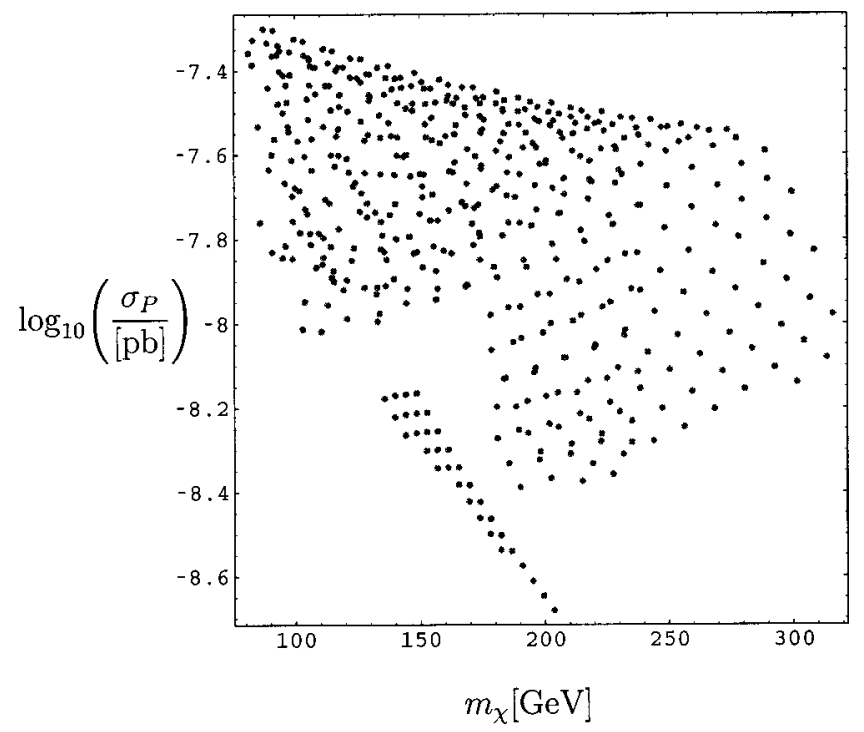

FIG. 9. Proton- $\chi$ cross section in the MSUGRA scenario for the dark shaded region in Fig. 8, also with the chargino mass bound and the constraint from $b \rightarrow s \gamma$.

$b \rightarrow s \gamma$ from the CLEO experiment, $B\left(B \rightarrow X_{s} \gamma\right)>2$ $\times 10^{-4}$. Second, the allowed region is significantly widened and even the region with the $B$-ino-like LSP (the region for $m_{0} \lesssim 1 \mathrm{TeV}$ ) can lead to the desired mass density of dark matter. This is because, in the case of large $\tan \beta$, the annihilation cross section through the process $\chi \chi \rightarrow A \rightarrow f \bar{f}$ is strongly enhanced. The reason is that the coupling with fermions $A f \bar{f}$ is proportional to $\tan \beta$ and that the mass of the pseudo-Higgs boson $m_{A}$ is significantly reduced by large down-type Yukawa couplings, which results in a much smaller suppression factor $\left(m_{A} / m_{\chi}\right)^{4}$.

Here, we should add a comment. One may think that the formation of $Q$ balls does not occur in the focus point region, since a large soft mass squared $m_{0}^{2}$ would lead to the $K$-factor in Eq. (33) being positive. However, even if $m_{0}^{2}$ $=\mathcal{O}(10 \mathrm{TeV})$, the $K$-factor is negative as long as we adopt

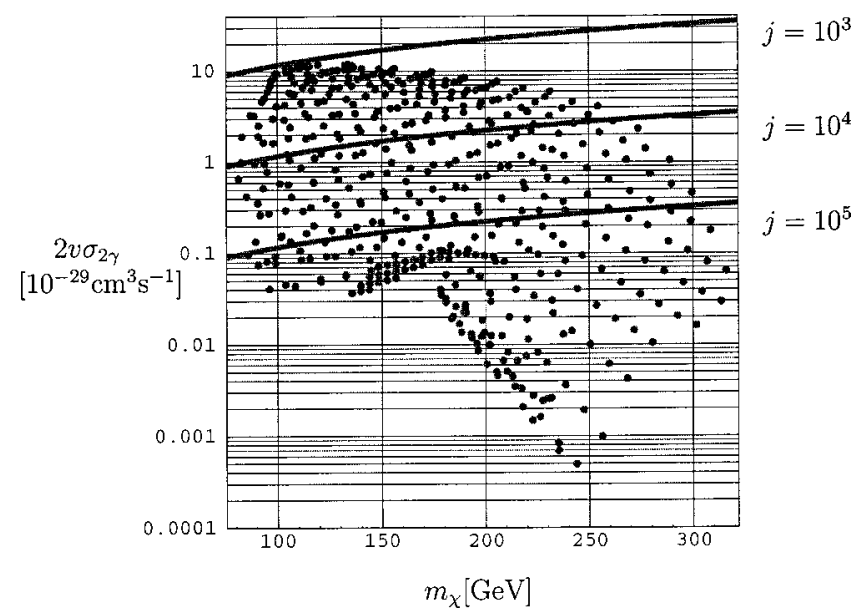

FIG. 10. Annihilation rate of neutralinos into the $2 \gamma$ final state in the MSUGRA scenario for the dark shaded region in Fig. 8, also with the chargino mass bound and the constraint from $b \rightarrow s \gamma$. 
flat directions, which are solely constructed by the first and second families. This condition can easily be satisfied in most of the flat directions.

\section{B. Parameter space and possibility of detection in the anomaly-mediation model}

In pure anomaly-mediated SUSY breaking, soft terms are determined by RG-invariant expressions involving the gauge and Yukawa couplings; hence the soft terms are completely fixed by the low energy values of these couplings and an overall scale $m_{3 / 2}$ :

$$
\begin{aligned}
& M_{\lambda}=-\frac{g^{2}}{2} \frac{d g^{-2}}{d \ln \mu} m_{3 / 2}=\frac{\beta_{g}}{g} m_{3 / 2} \\
& m_{\tilde{\Phi}}^{2}=-\frac{1}{4} \frac{d^{2} \ln Z_{\Phi}}{d(\ln \mu)^{2}} m_{3 / 2}^{2}=-\frac{1}{4}\left(\frac{\partial \gamma}{\partial g} \beta_{g}+\frac{\partial \gamma}{\partial y} \beta_{y}\right) m_{3 / 2}^{2} \\
& A_{y}=\frac{1}{2} \sum_{i} \frac{d \ln Z_{\Phi}}{d \ln y} m_{3 / 2}=-\frac{\beta_{y}}{y} m_{3 / 2}
\end{aligned}
$$

where $g, y$ are gauge and Yukawa couplings, respectively, $\Phi$ denotes the general superfield, and $m_{\Phi}^{2}, Z_{\Phi}$ are its soft scalar mass squared and wave function renormalization factor; $M_{\lambda}$ is the soft mass for the gaugino $\lambda, A_{y}$ is the trilinear scalar coupling associated with the Yukawa interaction with a coupling constant $y$. Here the sum $\Sigma_{i}$ runs through the fields included in this Yukawa interaction. We have defined the renormalization group functions as $\gamma(g, y) \equiv d \ln Z / d \ln \mu$, $\beta_{g}(g, y) \equiv d g / d \ln \mu$, and $\beta_{y} \equiv d y / d \ln \mu$.

As you can see from these relations, the soft masses squared for sleptons are negative, which is the biggest difficulty in pure AMSB models. Although many possible solutions are proposed [62], we adopt, in the present work, a phenomenological solution to the negative slepton mass problem. We assume the additional universal scalar mass $m_{0}$ at the GUT scale,

$$
m_{\Phi}^{2}=-\frac{1}{4}\left(\frac{\partial \gamma}{\partial g} \beta_{g}+\frac{\partial \gamma}{\partial y} \beta_{y}\right) m_{3 / 2}^{2}+m_{0}^{2}
$$

and then evolve the RG equations to obtain the low energy spectrum. Then the entire parameter space is specified by the following 4 parameters in this minimal framework:

$$
m_{3 / 2}, m_{0}, \tan \beta, \operatorname{sgn}(\mu) .
$$

From Eq. (81), we see that the ratios between the gaugino masses at the weak scale are approximately given by

$$
M_{1}: M_{2}: M_{3} \approx 2.8: 1:-8.3 \text {, }
$$

where $M_{1}, M_{2}$ and $M_{3}$ are the gaugino masses for $\mathrm{U}(1)_{Y}$, $\mathrm{SU}(2)_{L}$ and $\mathrm{SU}(3)_{C}$, respectively. Therefore, in the present scenario, the $\widetilde{W}$ LSP is realized in most of the parameter space.

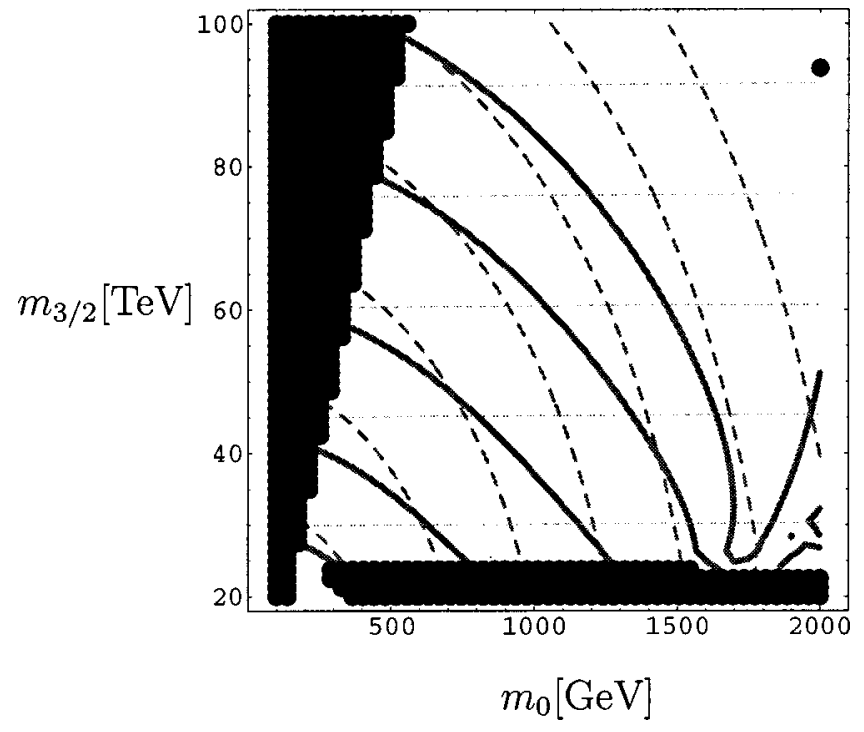

FIG. 11. Allowed parameter space and proton- $\chi$ cross section in the anomaly-mediation model with $\tan \beta=15$. Here, we take $\operatorname{sgn}(\mu)$ negative. In this plot, there is no region excluded by $b$ $\rightarrow s \gamma$. The black shaded region is excluded by $\tilde{\tau}$-LSP or the produced neutralinos overclose the universe. The wide white region leads to a desired mass density of dark matter via decays of $Q$ balls. The thick lines are contours of the proton- $\chi$ cross section, which are $\sigma_{P}=10^{-8}, 10^{-9}, 10^{-10}, 10^{-11}$ and $10^{-12} \mathrm{pb}$, from left to right, respectively. The dashed lines are contours of the mass of the heavy Higgs boson $m_{H}$, which are, from left to right, $500,750,1000, \ldots 2000 \mathrm{GeV}$. The dotted lines are contours of the lightest neutralino mass $m_{\chi}$ for $100,150, \ldots 300 \mathrm{GeV}$.

In Fig. 11, we present a parameter space where the LSPs produced by late-time decays of $Q$ balls provide a desired mass density of dark matter and the proton- $\chi$ scalar cross section in that region. Here we take $\tan \beta=15$, and $\operatorname{sgn}(\mu)$ negative to avoid too large a branching ratio of $b \rightarrow s \gamma$. There is no region excluded from the constraints on $b$ $\rightarrow s \gamma$. The black shaded region is excluded by the $\tilde{\tau}$ LSP and/or overclosing universe by the LSPs produced via $Q$-ball decays. In all of the remaining white space, the $\widetilde{W}$ LSP from the $Q$ ball decay can be the dominant component of the dark matter. The typical decay temperature of $Q$ balls resulting in the suitable mass density of dark matter is $10 \mathrm{MeV} \leq T_{d}$ $\lesssim 100 \mathrm{MeV}$. On the other hand, the mass splitting between the charged and neutral $\widetilde{W}$ 's is of order $100 \mathrm{MeV}$ to $1 \mathrm{GeV}$ $[63,64]$, which allows us to neglect the co-annihilation effects.

We present contours of the proton- $\chi$ cross section by the thick lines, which correspond to $\sigma_{P}$ $=10^{-8}, 10^{-9}, \ldots, 10^{-12} \mathrm{pb}$ from left to right, respectively. A strange structure appearing in the lower right-hand corner indicates a contamination of the $\widetilde{H}$ component in the LSP. The dotted lines are the contours of the lightest neutralino mass, $m_{\chi}=100,150, \ldots, 300 \mathrm{GeV}$, respectively. We also present the contours of heavy Higgs boson mass by the dashed lines, which denote $m_{H}=500,750, \ldots, 2000 \mathrm{GeV}$ from left to right, respectively. In contrast to the previous expectation $[2,65]$, the direct detection rates are rather small, 


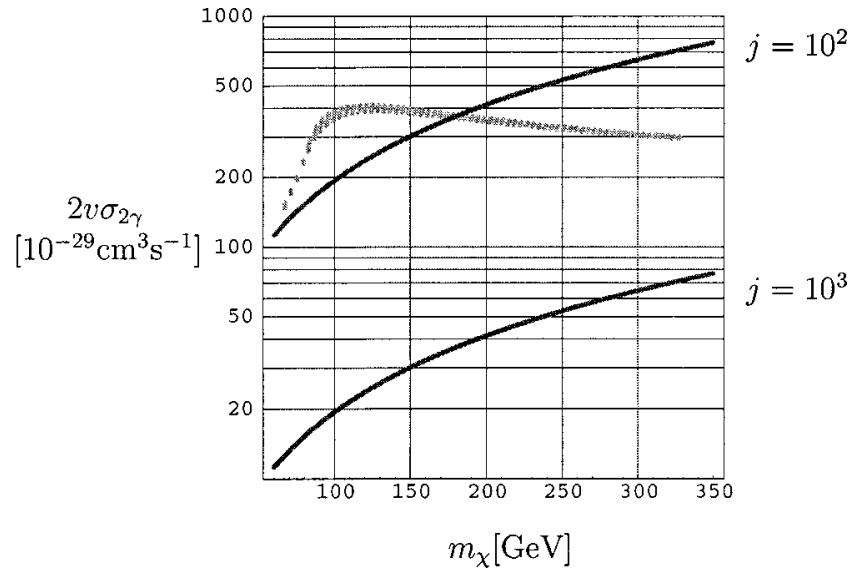

FIG. 12. Annihilation rate of neutralinos into the $2 \gamma$ final state in the AMSB model with $\tan \beta=15$. Each dot corresponds to one parameter set in the white region in Fig. 11. The two solid lines denote the $5 \sigma$ sensitivity curves for $j=10^{2}, 10^{3}$.

and only a restricted region is within the reach of the nextgeneration detectors, $\sigma_{P} \gtrsim 10^{-(9-8)} \mathrm{pb}$. This comes from the large mass of the heavy Higgs boson $m_{H}$. In relatively large $\tan \beta$, the proton- $\chi$ cross section is dominated by $H$ exchange, and it reduces as $\sigma_{P} \propto m_{H}^{-4}$. In Refs. [2,65], it was assumed that $m_{H} \simeq 300 \mathrm{GeV}$, which is realized only in a small space, as one can see from Fig. 11.

Fortunately, we may obtain a clear signal from the neutralino annihilation into the $2 \gamma$ final state [66]. In Fig. 12, we show the annihilation rate of the LSP into the $2 \gamma$ final state. Each dot falls in somewhere in the allowed space presented in Fig. 11. The solid lines are $5 \sigma$ sensitivity curves for $j$ $=10^{2}$ and $10^{3}$, which are estimated by the same parameter set as was used in Figs. 7 and 10, but we do not assume any improvements on the hadronic rejection and use $\epsilon_{\text {had }}=1$ in Ref. [59]. Even if we do not assume strongly cuspy profiles of the dark matter density in the halo, a large portion of the parameter space predicts a measurable size of the $\gamma$-ray flux.

In Figs. 13 and 14, we show the plots for the case of $\tan \beta=30$ and $\operatorname{sgn}(\mu)<0$. Conventions are almost the same as those in Figs. 11 and 12, respectively, but they are explicitly denoted in the caption to each figure. In such a large $\tan \beta$, we can see that the proton- $\chi$ cross section is strongly enhanced. This is because the $H$-nucleon-nucleon coupling, which dominates the proton- $\chi$ cross section, is proportional to $\tan \beta$, and the large down-type Yukawa couplings reduce the mass of the heavy Higgs boson $m_{H}^{2}$. We can expect the direct detection of the $\widetilde{W}$ dark matter in a relatively wide parameter space in the next generation of detectors. In Fig. 13 , a small region with $m_{0} \lesssim 500 \mathrm{GeV}, m_{3 / 2} \leqslant 30 \mathrm{TeV}$ is excluded by the constraint $B\left(B \rightarrow X_{s} \gamma\right)>2 \times 10^{-4}$ [67], although it is not depicted in the figure. The neutralino annihilation rate into the $2 \gamma$ final state is almost the same as in the case of $\tan \beta=15$, which is consistent with the result of Ref. [66].

In AMSB models, the neutralino annihilation into the $Z \gamma$ final state is known to be nearly twice that into the $2 \gamma$ final state (see Ref. [66]). Note that, in such a minimal framework

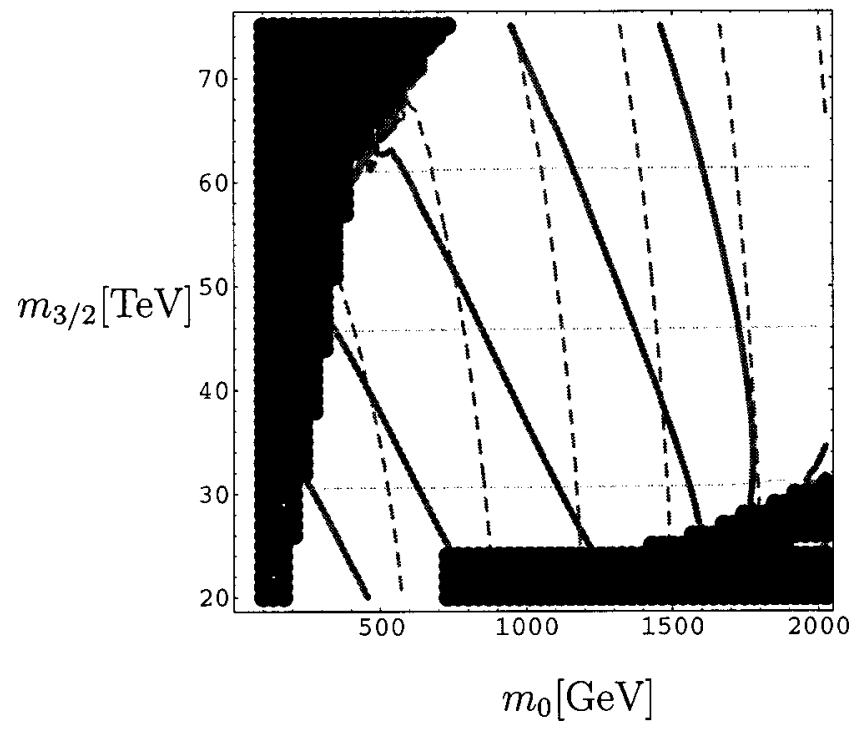

FIG. 13. Allowed parameter space and the proton- $\chi$ cross section in the anomaly-mediation model with $\tan \beta=30$. Here, we take $\operatorname{sgn}(\mu)$ negative. The black shaded region is excluded by $\tilde{\tau}$-LSP or the produced neutralinos overclose the universe. The black region also includes small regions where we cannot obtain the convergence of SOFTSUSY code. The wide white region leads to a desired mass density of dark matter via decays of $Q$ balls. The thick lines are contours of the proton- $\chi$ cross section, which are $\sigma_{P}$ $=10^{-7}, 10^{-8}, 10^{-9}, 10^{-10}$ and $10^{-11} \mathrm{pb}$, from left to right, respectively. The dashed lines are contours of the mass of the heavy Higgs boson $m_{H}$, which are, from left to right, $500,750,1000, \ldots 1750 \mathrm{GeV}$. The dotted lines are contours of the lightest neutralino mass $m_{\chi}$ for $100,150,200 \mathrm{GeV}$.

of AMSB models, the thermal relic of the $\widetilde{W}$ cannot be a significant component of dark matter, because of its large annihilation cross section. Such a large annihilation cross section of the LSP is, on the contrary, much more advantageous for the $\mathrm{AD}$ baryogenesis with late-time decays of $Q$ balls, although the conditions given in Eq. (31) or (32) should be satisfied.

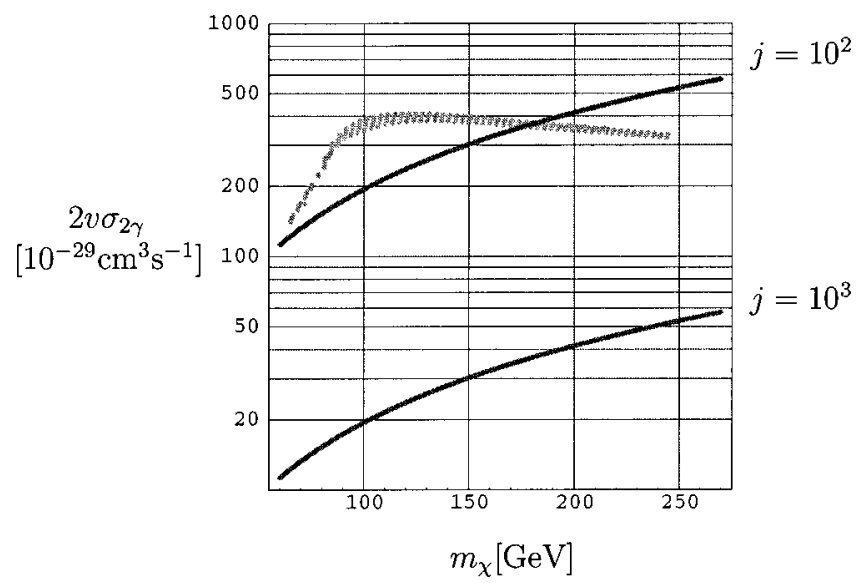

FIG. 14. Annihilation rate of neutralinos into the $2 \gamma$ final state in the AMSB model with $\tan \beta=30$. Conventions are the same as those in Fig. 12. 


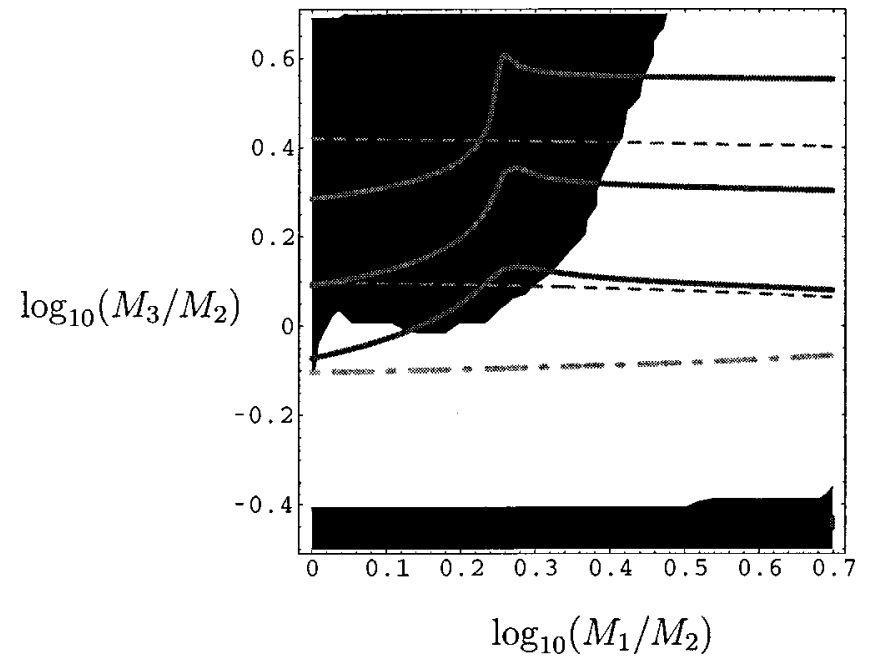

FIG. 15. Allowed region and the proton- $\chi$ scalar cross section $\sigma_{P}$ in the no-scale model with nonuniversal gaugino masses with $\tan \beta=10$. Here, we take $M_{2}=200 \mathrm{GeV}$ at the GUT scale. The thick lines are the contours of the $\sigma_{P}=10^{-7}, 10^{-8}, 10^{-9} \mathrm{pb}$ from the bottom up, respectively. The black shaded region is excluded by the fact that $\tilde{\tau}$ is the LSP, or the resultant LSPs from late-time decays of $Q$ balls overclose the universe, or the EWSB cannot be implemented. The dashed lines are the contours of the Higgs boson mass $m_{h}=114.1,120 \mathrm{GeV}$ and the region below the lower line is excluded. The region below the dot-dashed line is excluded since $B\left(B \rightarrow X_{s} \gamma\right)<2 \times 10^{-4}$.

\section{Parameter space and possibility of detection in no-scale models with nonuniversal gaugino masses}

In no-scale models [68], all the soft parameters except gaugino masses are assumed to vanish at some high energy scale $M_{X}$, which is usually taken to be the GUT scale $M_{X}$ $=M_{G}$. The soft parameters at the weak scale are generated by RG effects dominated by the gaugino masses, which are automatically flavor-blind and naturally suppress the SUSY flavor-changing neutral current (FCNC) interactions [69]. Recently, models with the no-scale boundary condition have attracted much attention, since a natural realization was found as gaugino-mediation models [70].

Unfortunately, the MSSM with the no-scale boundary condition at the GUT scale was found to be inconsistent with phenomenological requirements $[9,71,72]$. This is mainly due to the lower bound on the light Higgs boson mass and the cosmological requirement that a charged particle must not be the LSP. Several solutions were proposed to this problem, which are, imposing the no-scale boundary conditions above the GUT scale [73], gauging the $\mathrm{U}(1)_{B-L}$ symmetry [74] and assuming the nonuniversal gaugino masses at the GUT scale [9].

In the present work, we pick up the last scenario with nonuniversal gaugino masses. In this scenario, as investigated in Ref. [9], the $\widetilde{W}$ LSP is realized in a wide parameter space, which is expected to derive a desired mass density of dark matter from late-time decays of $Q$ balls.

In Fig. 15, we present allowed regions where the resultant LSPs give a desired mass density of dark matter and the proton- $\chi$ cross section in that region. Here, we take $M_{2}$

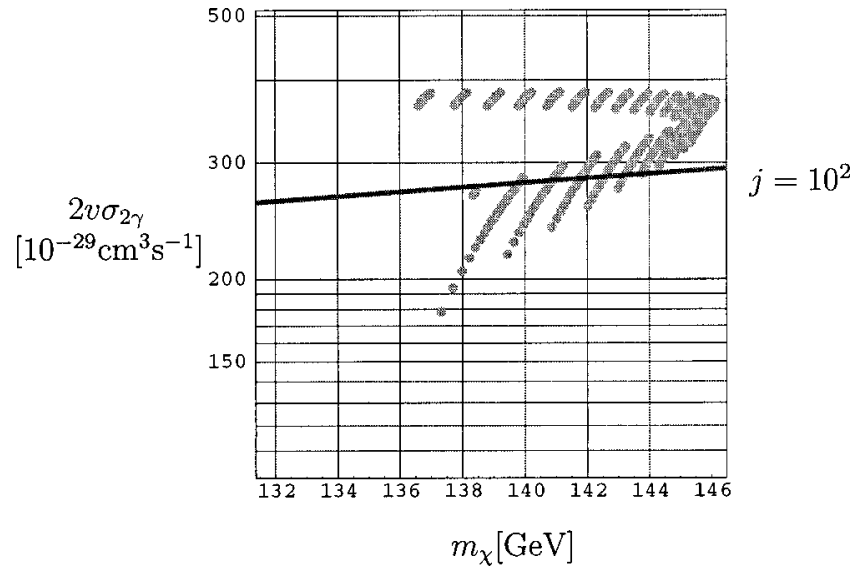

FIG. 16. Annihilation rate of neutralinos into the $2 \gamma$ final state for the allowed region in Fig. 15. Conventions are the same as those in Fig. 12.

$=200 \mathrm{GeV}, \tan \beta=10$ and vary the ratios of the gaugino masses at the GUT scale $M_{1} / M_{2}$ and $M_{3} / M_{2}$, which are denoted by the $x$-axis and the $y$-axis, respectively. The thick solid lines are the contours of the proton- $\chi$ cross section $\sigma_{P}$, which are $\sigma_{P}=10^{-7}, 10^{-8}$ and $10^{-9} \mathrm{pb}$ from the bottom up. The black shaded region is excluded, since the $\tilde{\tau}$ is the LSP, or the resultant LSPs overclose the universe, or the EWSB cannot be implemented. The dashed lines are the contours of the Higgs boson mass, $m_{h}=114.1$ and $120 \mathrm{GeV}$, and the region below the lower line is excluded by the Higgs boson mass bound [75]. The region below the dot-dashed line is excluded by the constraints on the $b \rightarrow s \gamma, B\left(B \rightarrow X_{s} \gamma\right)>2$ $\times 10^{-4}$.

In Fig. 16, we show the neutralino annihilation cross section into the $2 \gamma$ final state for the allowed region in Fig. 15. We also present the $5 \sigma$ sensitivity curve for $j=10^{2}$. Conventions are the same as those in Fig. 12 for anomaly-mediation models. In the allowed region, the LSP is given by the $\widetilde{W}$-like neutralino, which predicts large annihilation cross sections comparable with those in anomaly-mediation models.

In Figs. 17 and 18, we present the corresponding figures in the case of $\tan \beta=30$. Conventions are the same as those in Figs. 15 and 16. $\left(M_{2}=200 \mathrm{GeV}\right.$ is assumed at the GUT scale.) In this case, the direct detection rate is enhanced by large $\tan \beta$, but a large portion of the parameter space is excluded by the constraint from the $b \rightarrow s \gamma$. The $B$-ino-like LSP is realized in the small spot appearing on the left of the excluded region. In this region, the large annihilation cross section required from the late-time decays of $Q$ balls is provided by the pseudo-Higgs $A$ exchange diagram via the small $\widetilde{H}$ contamination in the LSP, whose amplitude is enhanced by the large $\tan \beta=30$ and the relatively small $m_{A}$. In this $B$-ino-like LSP region, the annihilation rate into the $2 \gamma$ final state is relatively small, $2 v \sigma_{2 \gamma} \sim 3 \times 10^{-29} \mathrm{~cm}^{3} \mathrm{sec}^{-1}$.

\section{Warm dark matter production from late-time decays of $Q$ balls}

Before closing this section, we should remark on an interesting possibility that the resultant $\widetilde{H}$-like LSPs (or maybe 


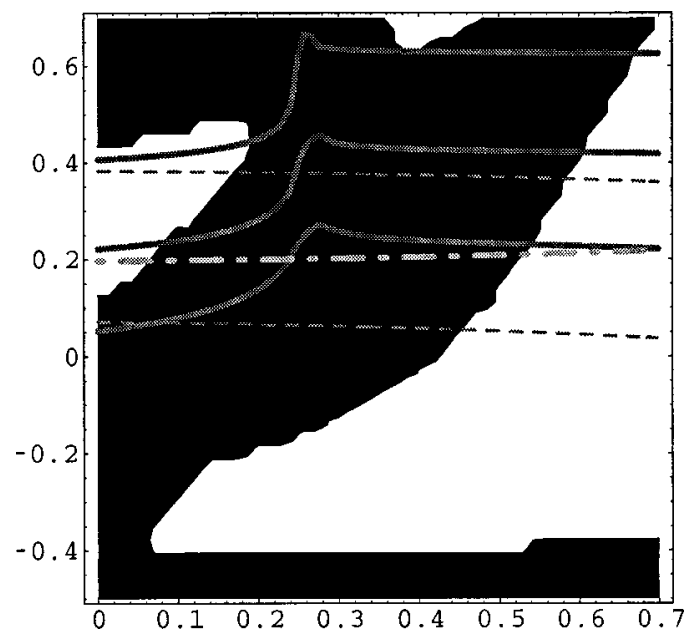

FIG. 17. Allowed region and the proton- $\chi$ scalar cross section $\sigma_{P}$ in the no-scale model with nonuniversal gaugino masses with $\tan \beta=30$. Conventions are the same as those in Fig. 15 . The $B$-inolike LSP is realized in the small spot appearing on the left of the excluded region. The required large annihilation cross section is obtained by the $A$ exchange diagram enhanced by the large $\tan \beta$ and the relatively small $m_{A}$.

$\widetilde{B}$-like LSP in the case of a large $\tan \beta$ ) form the warm dark matter (WDM). As we have seen, cuspy structures in the halo profiles have a drastic consequence on the future observations in indirect dark matter searches. However, it is argued that the neutralino cuspy profile might be inconsistent with the radio emission from the center of the Galaxy [76], although further detailed studies are needed to fix the situation. Maybe the inflation models with a suppressed strength in the small-scale perturbations might solve the problem. Another possible solution is the WDM.

If the neutralino LSPs are produced after the decoupling of thermal interactions with relativistic velocity, they may serve as a WDM and wash out the cuspy profiles in the halo $[77,78]$. The conditions needed for the MSSM neutralino to

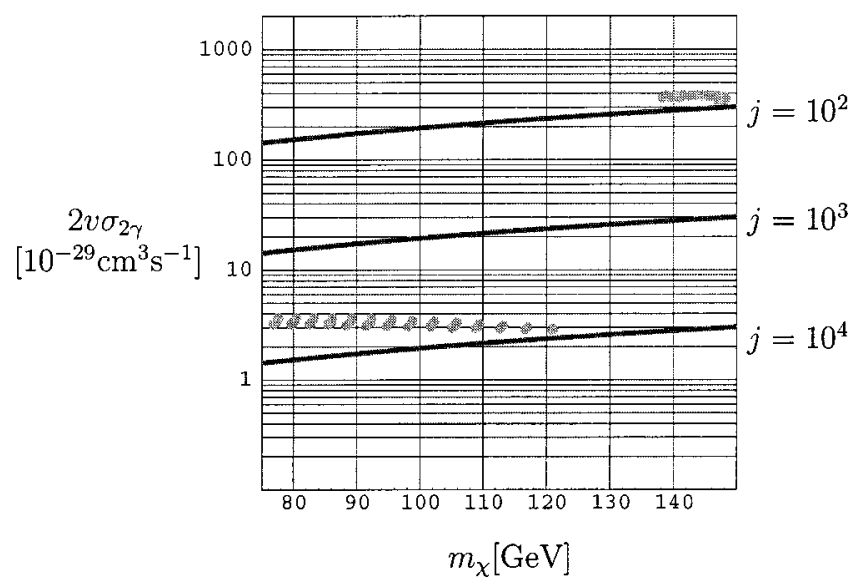

FIG. 18. Annihilation rate of neutralinos into the $2 \gamma$ final state for the allowed region in Fig. 17. Conventions are the same as those in Fig 12. The dots with smaller annihilation rates correspond to the region with the $B$-ino-like LSP. form a WDM were studied in Ref. [40]. Actually, these authors found that it is possible if the temperature is low enough when the neutralinos are produced via decays of nonthermal sources:

$$
T_{d} \lesssim 5 \mathrm{MeV} \text { for } \widetilde{B} \text {-like LSP }
$$

and

$$
T_{d} \lesssim 2 \mathrm{MeV} \text { for } \widetilde{H} \text {-like LSP. }
$$

As for the $\widetilde{W}$ LSP, they found it is impossible to form the WDM. If the above condition is satisfied, energy reductions of neutralinos by scatterings with thermal background are small enough. Such a situation may arise in some models, for example, the late-time decays of the heavy moduli $[79,65]$ and the evaporation of cosmological defects [78]. Here, we propose another way to generate WDM via the late-time decays of $Q$ balls.

To obtain the sufficient free streaming length needed to suppress the small-scale perturbations, the current velocity of the LSP should satisfy [78]

$$
v_{0} \simeq 10^{-(7-8)} \text {. }
$$

If neutralinos are produced via late-time decays of $Q$ balls, the current velocity of the neutralinos is given by

$$
v_{0}=\frac{T_{0}}{T_{d}} \frac{m_{\phi}}{m_{\chi}} \simeq 2.4 \times 10^{-8}\left(\frac{1 \mathrm{MeV}}{T_{d}}\right)\left(\frac{m_{\phi}}{10 \mathrm{TeV}}\right)\left(\frac{100 \mathrm{GeV}}{m_{\chi}}\right),
$$

where $T_{0}=2.7 \mathrm{~K}$ is the current temperature of the cosmic microwave background and $T_{d}$ is the decay temperature of $Q$ balls; $m_{\phi}$ is the mass of the relevant flat direction field $\phi$. Here, we have assumed that there is no substantial energy reduction by scattering with thermal backgrounds, which is justified if the condition in Eq. (85) is satisfied.

Therefore the resultant neutralinos from late-time decays of $Q$ balls may serve as a WDM, for example, in "focus point supersymmetry" with $m_{0}=\mathcal{O}(10 \mathrm{TeV})$ or in "effective SUSY models" with very large soft masses for the squarks in the first and second families $m_{\widetilde{Q}_{1,2}}^{2}=\mathcal{O}(10 \mathrm{TeV})^{2}$ [80].

\section{CONCLUSIONS AND DISCUSSIONS}

Both of the production mechanisms of the observed baryon asymmetry and the natures of dark matter are among the most fundamental problems in particle physics as well as in cosmology. In the SUSY framework, there exist rather stringent upper bounds on reheating temperatures of inflation because of the "cosmological gravitino problem," which strongly constrains various baryo/leptogenesis scenarios requiring relatively high reheating temperatures. Affleck-Dine baryogenesis is one of the most promising candidates to generate the observed baryon asymmetry in low reheating temperatures, which is free from the gravitino problem.

In recent developments, it has become clear that the formation of $Q$ balls and their late-time decays are almost in- 
evitable consequences of AD baryogenesis. ${ }^{13}$ This requires a significantly large annihilation cross section of the LSP for the resultant LSPs not to overclose the universe.

In this paper, we investigated in detail the predictions of the late-time decays of $Q$ balls. First, we reviewed the AD baryogenesis, the $Q$ ball formation and decays, with thermal effects taken into account. Second, we discussed the nonthermal production of dark matter via the late-time decays of $Q$ balls with and without entropy production. Finally, we specified the allowed parameter space where the neutralinos produced via the decays of $Q$ balls result in a desired mass density of the dark matter in several SUSY breaking models, and then we discussed the prospects of direct and indirect detection of these nonthermal dark matter candidates. We also discussed the possibility of WDM generation via $Q$ ball decays.

As an interesting new twist, we pointed out that the $\mathrm{AD}$ baryogenesis without a superpotential is now one of the most beautiful scenarios. Quite a reasonable set of parameters can explain the observed baryon asymmetry without any assumptions on additional late-time entropy productions, such as the decay of heavy moduli or a thermal inflation. The resultant baryon asymmetry and dark matter density are determined solely by the potential for the AD field and the annihilation cross section of the LSP. They are completely independent of the reheating temperature of the inflation and any other details in the history of the universe.

The parameter space and detection possibility for each SUSY breaking model can be summarized as follows:

(i) In minimal supergravity scenarios, allowed parameter space appears in the "focus-point" region where the dominant component of the LSP is provided by $\widetilde{H}$. A large content of $\widetilde{H}$ in the LSP strongly enhances the direct detection rate through the Higgs exchange diagrams. As a result, the direct detection rate is within the reach of various next-generation detectors in most of the parameter space. The detection rate of the monoenergetic $\gamma$-rays via neutralino annihilation is also enhanced with respect to $B$-ino-like dark matter, especially in pure $\widetilde{H}$ LSP regions. Somewhat cuspy density profiles of the halo $j \geq 10^{3.5}$ allow us to observe the monoenergetic $\gamma$-ray lines in the case of pure $\widetilde{H}$ dark matter.

(ii) In anomaly-mediated SUSY breaking models, the LSP is $\widetilde{W}$-like in most of the parameter space, which is now a promising candidate of the dark matter because of the latetime decays of $Q$ balls. The direct detection rate highly depends on $\tan \beta$ and becomes very large in the case of large $\tan \beta$. This is because the coupling of the heavy Higgs boson with the nucleon is proportional to $\tan \beta$, and also the mass of the heavy Higgs boson is strongly reduced by the large down-type Yukawa couplings. The indirect detection rate using monoenergetic $\gamma$-rays is surprisingly large, as reported in Ref. [66], and is almost independent of $\tan \beta$. Even if we do not assume a highly cuspy density profile, the detection rate

\footnotetext{
${ }^{13}$ This is not the case for the AD leptogenesis via the $L H_{u}$ flat direction. See Sec. II.
}

is within the reach of the next generation of air Cherenkov telescopes [49-51].

(iii) In no-scale models with nonuniversal gaugino masses, the LSP is $\widetilde{W}$-like in a wide parameter space. (The region with the $\widetilde{H}$ LSP is excluded by the Higgs mass bound and the constraints on the $b \rightarrow s \gamma$ branching ratio.) The direct and indirect detection rates are comparable with those in anomaly-mediation models, and within the reach of various next-generation detectors.

Although we have concentrated on the monoenergetic $\gamma$-rays for the indirect detection in this paper, the possibility of detecting the neutralino in other indirect dark matter searches is also significantly enhanced in the case of $\widetilde{H}$ and $\widetilde{W}$ dark matter. The prospects of other indirect detections in MSUGRA scenarios can be obtained in Refs. [81]. As for the anomaly-mediation models, see Refs. $[66,65]$. Last year, the HEAT experiment [82] confirmed an excess of a high energy positron flux in cosmic rays [83]. If the LSP is $\widetilde{H}$ - or $\widetilde{W}$-like, the annihilation into a pair of $W$ bosons always dominates as long as $m_{\chi}>m_{W}$. The subsequent decay of a $W$ boson into a positron, $W^{+} \rightarrow e^{+}+\nu$, will produce a large excess of positron flux at and below an energy of about half the $W$ boson mass. In fact, it was shown in Ref. [84] that the observed positron spectrum is naturally explained by the annihilation of neutralino dark matter, if it is $\widetilde{H}$ - or $\widetilde{W}$-like nonthermal dark matter. This fact may indicate the existence of nonthermal sources for these LSPs in the history of the universe.

Finally, we comment on some advantages of our scenario relative to the others that can produce the $\widetilde{H}$ or $\widetilde{W}$-like dark matter. Actually, there exist several other mechanisms that can generate the $\widetilde{H}$ - or $\widetilde{W}$-like nonthermal dark matter, which are the decays of heavy moduli fields and/or heavy gravitinos with a mass of the order of $100 \mathrm{TeV}$ [65], and the evaporations of topological defects [78]. The former case only appears in the anomaly-mediated SUSY breaking scenarios. Furthermore, the decays of heavy moduli fields are usually accompanied by an extra large entropy production, which causes a serious problem to explain the observed baryon asymmetry. If one relies on the $\mathrm{AD}$ baryogenesis to produce enough baryon asymmetry, then the resultant late-time $Q$ ball decays also play a role as the nonthermal source for the $\widetilde{W}$-like dark matter. As for the evaporation of topological defects, we have no clear reason to expect that they take place at the appropriate temperature of $1 \mathrm{MeV} \leq T_{d}$ $\lesssim$ (a few) $\mathrm{GeV}$, and that they produce enough initial abundance of the $\widetilde{H}$ - or the $\widetilde{W}$-like LSP to be a dominant component of dark matter. They may also cause a serious problem by diluting the baryon asymmetry.

In our scenario, the reasonable set of parameters in the scalar potential of the AD field to explain the observed baryon asymmetry naturally leads to the desirable decay temperature of the $Q$ balls for the nonthermal production of these LSPs in various SUSY breaking scenarios. The baryon asymmetry is, of course, guaranteed by the AD mechanism.

As we have seen in this article, $\widetilde{H}$ - and $\widetilde{W}$-like LSPs are now promising candidates for the dark matter. The possibility of direct and indirect detection of these dark matter can- 
didates is much larger than that of the standard $B$-ino-like LSP. If $\widetilde{H}$ or $\widetilde{W}$ dark matter is indeed confirmed in future experiments, it may shed a bright light on the origin of the whole matter in our universe.

Finally, we add a brief comment on the fine tunings on the soft SUSY breaking parameters to explain the mass density of dark matter for a given decay temperature of a $Q$ ball. As we have discussed in the text, the dominant annihilation channel of the Higgsino- and W-ino-like LSP is given by the decay channel into a pair of gauge bosons via the lightest chargino exchange. This means that the annihilation cross section of these LSPs is rather stable under the variation of other soft parameters, such as slepton and squark masses.
(Note that the variation of the squark masses has only a mild effect on the size of the $\mu$-term in the focus point region.) Consequently, no strong fine tuning on the soft SUSY breaking parameters is required for a given decay temperature of a $Q$ ball. This is a clear contrast to the standard pure $B$-ino dark matter, which needs a strong degeneracy between the $B$-ino and the lightest stau.

\section{ACKNOWLEDGMENTS}

M.F. and K.H. thank the Japan Society for the Promotion of Science for financial support.
[1] I. Affleck and M. Dine, Nucl. Phys. B249, 361 (1985).

[2] M. Fujii and K. Hamaguchi, Phys. Lett. B 525, 143 (2002).

[3] M. Dine, A.E. Nelson, and Y. Shirman, Phys. Rev. D 51, 1362 (1995); M. Dine, A.E. Nelson, Y. Nir, and Y. Shirman, ibid. 53, 2658 (1996); for a review, see, for example, G.F. Giudice and R. Rattazzi, Phys. Rep. 322, 419 (1999).

[4] G.R. Dvali, A. Kusenko, and M.E. Shaposhnikov, Phys. Lett. B 417, 99 (1998).

[5] A. Kusenko and M.E. Shaposhnikov, Phys. Lett. B 418, 46 (1998).

[6] K. Enqvist and J. McDonald, Phys. Lett. B 425, 309 (1998).

[7] K. Enqvist and J. McDonald, Nucl. Phys. B538, 321 (1999).

[8] L. Randall and R. Sundrum, Nucl. Phys. B557, 79 (1999); G.F. Giudice, M.A. Luty, H. Murayama, and R. Rattazzi, J. High Energy Phys. 12, 027 (1998); J.A. Bagger, T. Moroi, and E. Poppitz, ibid. 04, 009 (2000).

[9] S. Komine and M. Yamaguchi, Phys. Rev. D 63, 035005 (2001).

[10] J.L. Feng, K.T. Matchev, and T. Moroi, Phys. Rev. Lett. 84, 2322 (2000); J.L. Feng, K.T. Matchev, and T. Moroi, Phys. Rev. D 61, 075005 (2000).

[11] L. Bergstrom and P. Ullio, Nucl. Phys. B504, 27 (1997).

[12] M. Dine, L. Randall, and S. Thomas, Phys. Rev. Lett. 75, 398 (1995); Nucl. Phys. B458, 291 (1996).

[13] T. Gherghetta, C. Kolda, and S.P. Martin, Nucl. Phys. B468, 37 (1996).

[14] V.A. Kuzmin, V.A. Rubakov, and M.E. Shaposhnikov, Phys. Lett. 155B, 36 (1985).

[15] M. Fukugita and T. Yanagida, Phys. Lett. B 174, 45 (1986).

[16] H. Murayama and T. Yanagida, Phys. Lett. B 322, 349 (1994).

[17] T. Moroi and H. Murayama, J. High Energy Phys. 07, 009 (2000).

[18] T. Asaka, M. Fujii, K. Hamaguchi, and T. Yanagida, Phys. Rev. D 62, 123514 (2000).

[19] M. Fujii, K. Hamaguchi, and T. Yanagida, Phys. Rev. D 63, 123513 (2001).

[20] M. Fujii, K. Hamaguchi, and T. Yanagida, Phys. Rev. D 65, 043511 (2002).

[21] M. Fujii, K. Hamaguchi and T. Yanagida, Phys. Lett. B 538, 107 (2002).

[22] R. Allahverdi, M. Drees, and A. Mazumdar, Phys. Rev. D 65, 065010 (2002).
[23] K. Enqvist, A. Jokinen, and J. McDonald, Phys. Lett. B 483, 191 (2000).

[24] M. Fujii, K. Hamaguchi, and T. Yanagida, Phys. Rev. D 64, 123526 (2001).

[25] R. Allahverdi, A. Mazumdar, and A. Ozpineci, Phys. Rev. D 65, 125003 (2002).

[26] R. Allahverdi, B.A. Campbell, and J.R. Ellis, Nucl. Phys. B579, 355 (2000).

[27] N. Sakai and T. Yanagida, Nucl. Phys. B197, 533 (1982); S. Weinberg, Phys. Rev. D 26, 287 (1982).

[28] T. Goto and T. Nihei, Phys. Rev. D 59, 115009 (1999).

[29] J. Hisano, H. Murayama, and T. Yanagida, Nucl. Phys. B402, 46 (1993).

[30] A. Anisimov and M. Dine, Nucl. Phys. B619, 729 (2001).

[31] M.Y. Khlopov and A.D. Linde, Phys. Lett. B 138, 265 (1984); J. Ellis, J.E. Kim, and D.V. Nanopoulos, ibid. 145B, 181 (1984); M. Kawasaki and T. Moroi, Prog. Theor. Phys. 93, 879 (1995); see also a recent analysis, M. Kawasaki, K. Kohri, and T. Moroi, Phys. Rev. D 63, 103502 (2001).

[32] M. Kawasaki, T. Watari, and T. Yanagida, Phys. Rev. D 63, 083510 (2001).

[33] S.R. Coleman, Nucl. Phys. B262, 263 (1985); B269, 744 (1985).

[34] S. Kasuya and M. Kawasaki, Phys. Rev. D 62, 023512 (2000).

[35] K. Enqvist and J. McDonald, Nucl. Phys. B570, 407 (2000).

[36] S. Kasuya and M. Kawasaki, Phys. Rev. D 64, 123515 (2001).

[37] M. Laine and M.E. Shaposhnikov, Nucl. Phys. B532, 376 (1998).

[38] R. Banerjee and K. Jedamzik, Phys. Lett. B 484, 278 (2000).

[39] A.G. Cohen, S.R. Coleman, H. Georgi, and A. Manohar, Nucl. Phys. B272, 301 (1986).

[40] J. Hisano, K. Kohri, and M.M. Nojiri, Phys. Lett. B 505, 169 (2001).

[41] V.A. Bednyakov, H.V. Klapdor-Kleingrothaus, and S. Kovalenko, Phys. Rev. D 50, 7128 (1994).

[42] G. Jungman, M. Kamionkowski, and K. Griest, Phys. Rep. 267, 195 (1996).

[43] R.W. Schnee et al., Phys. Rep. 307, 283 (1998).

[44] CRESST-Collaboration, M. Bravin et al., Astropart. Phys. 12, 107 (1999).

[45] G. Chardin, "Edelweiss dark matter search," talk given at the 
School and Workshop on Neutrino Particle Astrophysics, Les Houches, 2002.

[46] GENIUS Collaboration, H.V. Klapdor-Kleingrothaus et al., hep-ph/9910205.

[47] N. Spooner, "New limits and progress from the boulby dark matter program," talk given at the same workshop as in Ref. [45].

[48] P. Ullio and L. Bergstrom, Phys. Rev. D 57, 1962 (1998).

[49] VERITAS Collaboration, S. Bradbury et al., in Proc. of the 26th ICRC, Salt Lake City, 1999, OG. 4. 3. 28.

[50] HESS Collaboration, A. Kohnle et al., in Ref. [49], OG. 4. 3. 13.

[51] MAGIC Collaboraton, M. Martinez et al., in Ref. [49], OG. 4. 3. 08.

[52] M. Drees and M.M. Nojiri, Phys. Rev. D 47, 376 (1993).

[53] B.C. Allanach, Comput. Phys. Commun. 143, 305 (2002).

[54] J. Bagger, K.T. Matchev, and D. Pierce, Phys. Lett. B 348, 443 (1995); D.M. Pierce, J.A. Bagger, K.T. Matchev, and R.j. Zhang, Nucl. Phys. B491, 3 (1997).

[55] Joint LEP 2 Supersymmetry Working Group, Combined LEP Chargino Results, http://lepsusy.web.cern.ch/lepsusy/www/ inos_moriond01/charginos_pub.html.

[56] G. Degrassi, P. Gambino, and G.F. Giudice, J. High Energy Phys. 12, 009 (2000), and references therein.

[57] CLEO Collaboration, S. Ahmed et al., hep-ex/9908022; CLEO Collaboration, S. Chen et al., Phys. Rev. Lett. 87, 251807 (2001).

[58] M. Drees and M. Nojiri, Phys. Rev. D 48, 3483 (1993).

[59] L. Bergstrom, P. Ullio, and J.H. Buckley, Astropart. Phys. 9, 137 (1998).

[60] S. Ghigna, B. Moore, F. Governato, G. Lake, T. Quinn, and J. Stadel, Astrophys. J. 544, 616 (2000).

[61] J.F. Navarro, C.S. Frenk and S.D. White, Astrophys. J. 462, 563 (1996)

[62] L. Randall and R. Sundrum, in Ref. [8]; A. Pomarol and R. Rattazzi, J. High Energy Phys. 05, 013 (1999); E. Katz, Y. Shadmi, and Y. Shirman, ibid. 08, 015 (1999); D.E. Kaplan and G.D. Kribs, ibid. 09, 048 (2000); N. Arkani-Hamed, D.E. Kaplan, H. Murayama, and Y. Nomura, ibid. 02, 041 (2001); I. Jack and D.R. Jones, Phys. Lett. B 482, 167 (2000); A.E. Nelson and N.J. Weiner, Phys. Rev. Lett. 88, 231802 (2002); R. Harnik, H. Murayama, and A. Pierce, hep-ph/0204122.

[63] T. Gherghetta, G.F. Giudice, and J.D. Wells, Nucl. Phys. B559, 27 (1999).

[64] J.L. Feng, T. Moroi, L. Randall, M. Strassler, and S.f. Su,
Phys. Rev. Lett. 83, 1731 (1999).

[65] T. Moroi and L. Randall, Nucl. Phys. B570, 455 (2000).

[66] P. Ullio, J. High Energy Phys. 06, 053 (2001).

[67] J.L. Feng and T. Moroi, Phys. Rev. D 61, 095004 (2000).

[68] J.R. Ellis, C. Kounnas, and D.V. Nanopoulos, Nucl. Phys. B247, 373 (1984).

[69] K. Inoue, M. Kawasaki, M. Yamaguchi, and T. Yanagida, Phys. Rev. D 45, 328 (1992).

[70] D.E. Kaplan, G.D. Kribs, and M. Schmaltz, Phys. Rev. D 62, 035010 (2000); Z. Chacko, M.A. Luty, A.E. Nelson, and E. Ponton, J. High Energy Phys. 01, 003 (2000).

[71] S. Komine, hep-ph/0102030.

[72] J.R. Ellis, D.V. Nanopoulos, and K.A. Olive, Phys. Lett. B 525, 308 (2002).

[73] M. Schmaltz and W. Skiba, Phys. Rev. D 62, 095004 (2000).

[74] M. Fujii and K. Suzuki, Phys. Rev. D 66, 035002 (2002).

[75] LEP Higgs Working Group for Higgs boson searches Collaboration, "Search for the standard model Higgs boson at LEP," hep-ex/0107029; LEP Higgs Working Group Collaboration, "Searches for the neutral Higgs bosons of the MSSM: Preliminary combined results using LEP data collected at energies up to $209 \mathrm{GeV}$," hep-ex/0107030.

[76] P. Gondolo, Phys. Lett. B 494, 181 (2000).

[77] S. Hannestad, astro-ph/0008451.

[78] W.B. Lin, D.H. Huang, X. Zhang, and R.H. Brandenberger, Phys. Rev. Lett. 86, 954 (2001).

[79] J.R. Ellis, D.V. Nanopoulos, and M. Quiros, Phys. Lett. B 174, 176 (1986); T. Moroi, M. Yamaguchi, and T. Yanagida, ibid. 342, 105 (1995); G.F. Giudice, E.W. Kolb, and A. Riotto, Phys. Rev. D 64, 023508 (2001).

[80] M. Dine, A. Kagan, and S. Samuel, Phys. Lett. B 243, 250 (1990); S. Dimopoulos and G.F. Giudice, ibid. 357, 573 (1995); A. Pomarol and D. Tommasini, Nucl. Phys. B466, 3 (1996); A.G. Cohen, D.B. Kaplan, and A.E. Nelson, Phys. Lett. B 388, 588 (1996); J. Hisano, K. Kurosawa, and Y. Nomura, Nucl. Phys. B584, 3 (2000).

[81] J.L. Feng, K.T. Matchev, and F. Wilczek, Phys. Rev. D 63, 045024 (2001); V. Bertin, E. Nezri, and J. Orloff, hep-ph/0204135.

[82] S. Coutu et al., Proceedings of ICRC 2001.

[83] HEAT Collaboration, S.W. Barwick et al., Phys. Rev. Lett. 75, 390 (1995); Astrophys. J. Lett. 482, L191 (1997).

[84] G.L. Kane, L.T. Wang, and J.D. Wells, Phys. Rev. D 65, 057701 (2002); G.L. Kane, L.T. Wang, and T.T. Wang, Phys. Lett. B 536, 263 (2000). 